Sorghum halepense (L.) مقارنة فعالية بعض المبيدات العثبية في مكافحة عشبه الحليان

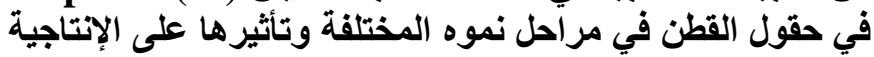

$$
\text { قسم وقاية النبات ـ كلية الزراعة الميمد - جامعة الفرات ـ سوريا }
$$

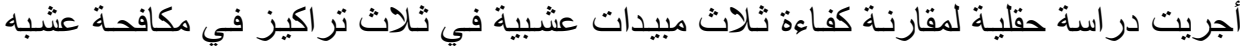

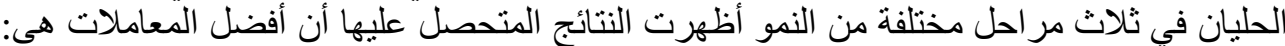

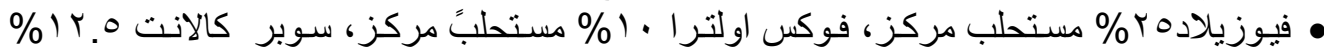

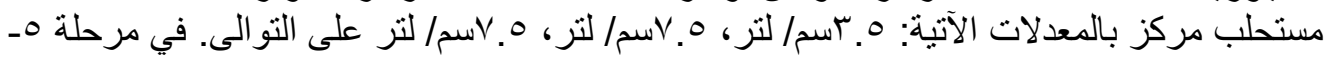

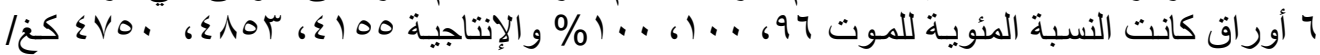

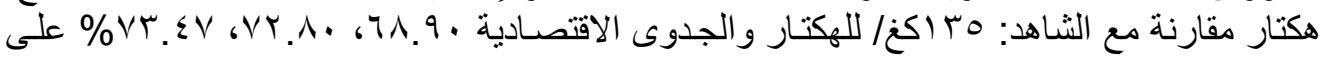

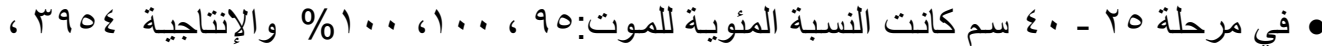

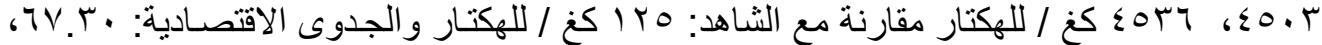

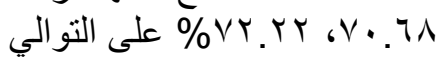

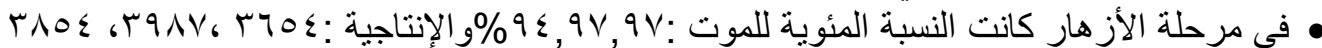

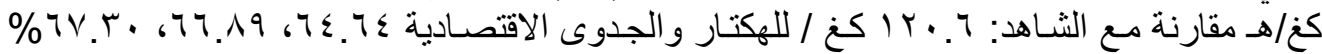

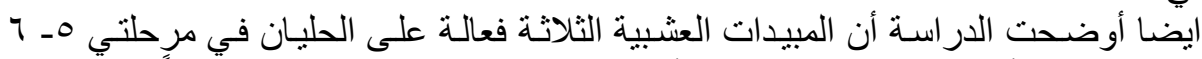

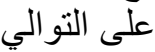

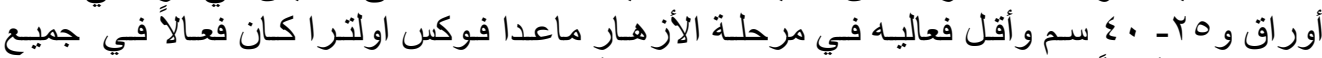

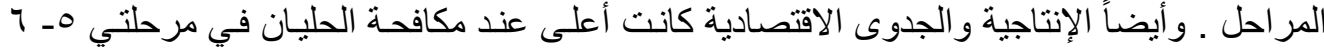

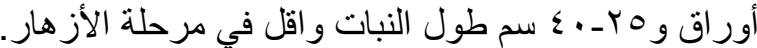
Sorghum الكلمات المفتاحية: المبيدات العشبية: فيوزبلاد، فوكس اولترا، سوبر كالات الانت ـ الحليان .halepense ( L .)

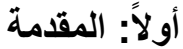

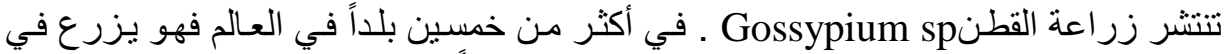

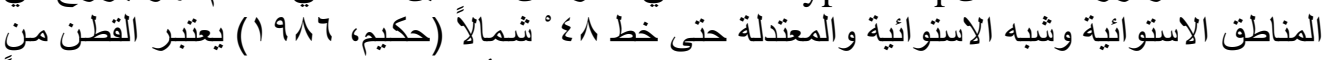

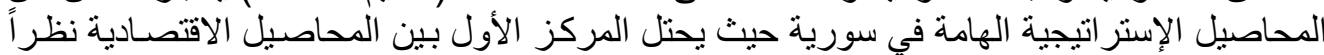

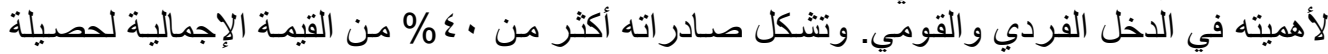
الصادر ات السورية (FA0, 1991 ).

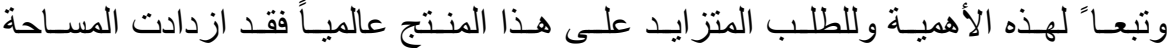

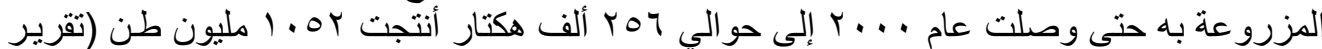

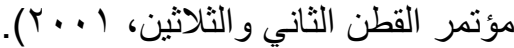

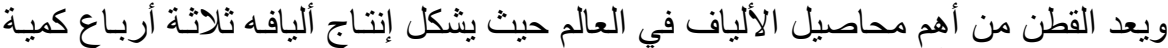

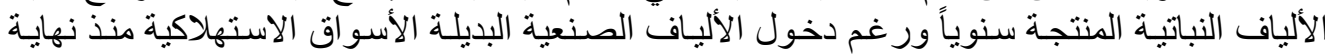

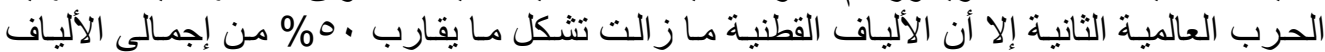

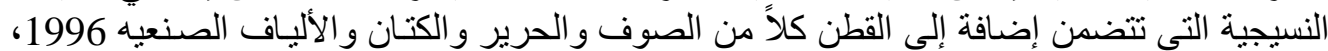

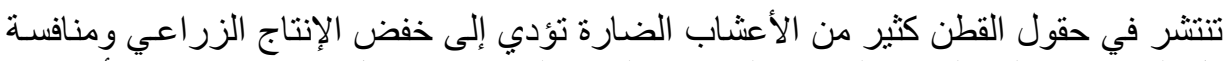

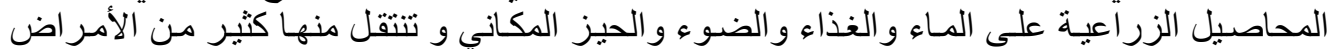

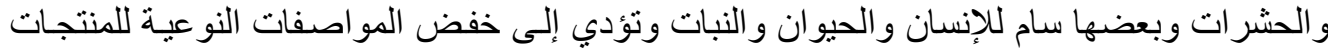
الغذائية و الحيوانية ومن هذه الأعثـاب الحليان Sorghum halepense (L.) فهو من أكثر الأنواع

Fayoum J. Agric. Res. \& Dev., Vol.22, No.2, July, 2008 


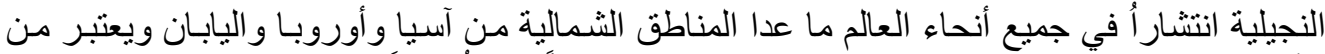

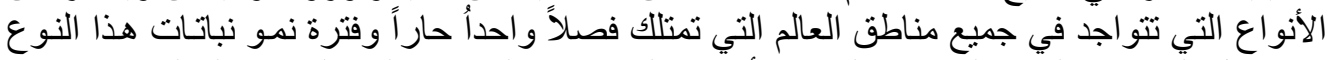

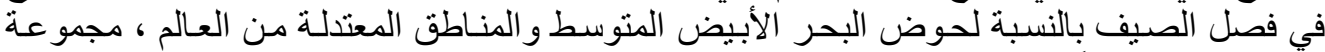

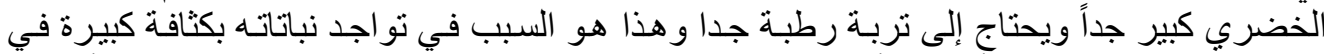

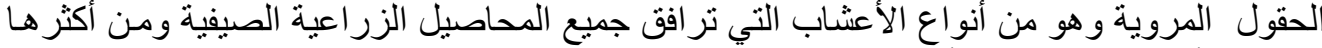

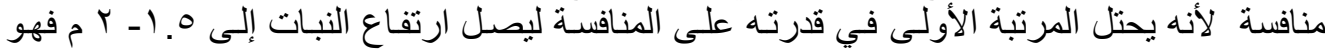

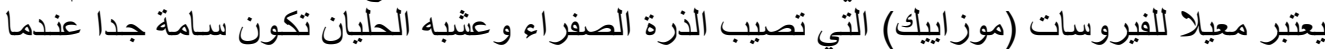

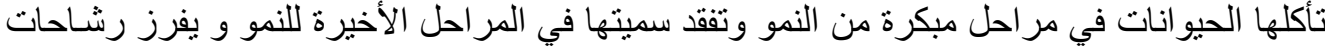

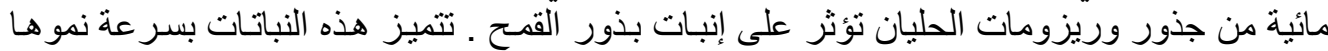

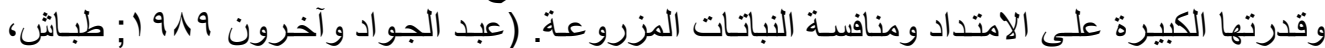
(199.

ثانياً: هدف البحث

يهدف البحث إلى مقارنة فعالية بعض المبات المبيدات العشبية على عشبه الحليان في مر احل نموها المختلفة وتأثير ها على إنتاجية القطن. ثالثاً: مواد وطرائق البحث عانث

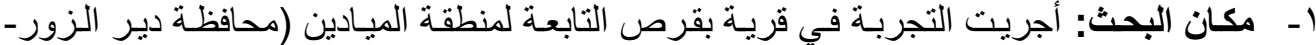

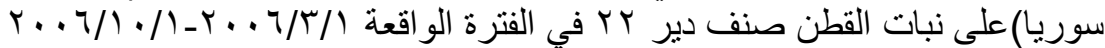
r

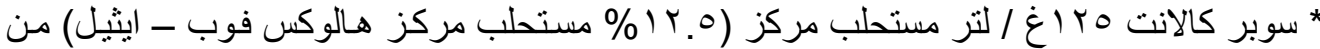

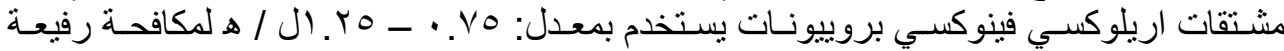

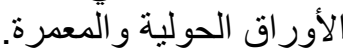

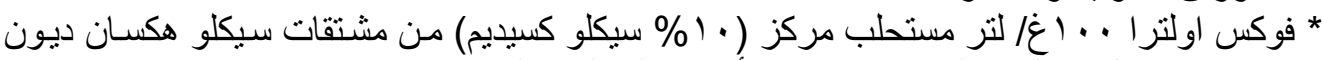

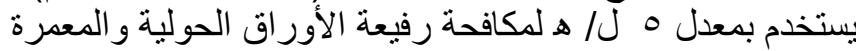

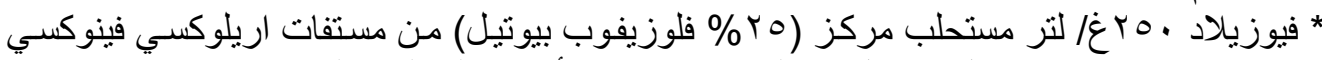

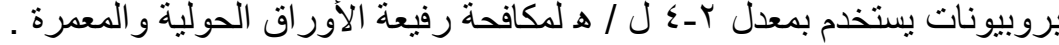

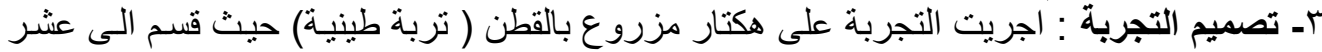

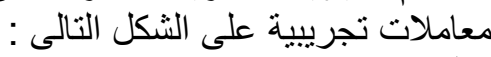

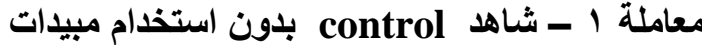

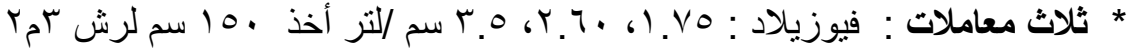

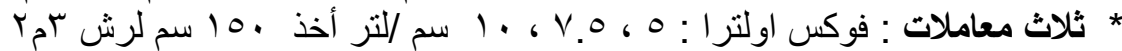

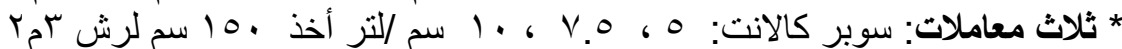

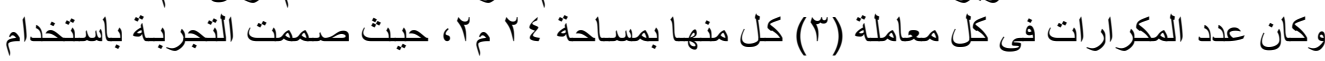

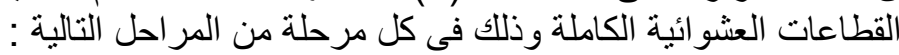

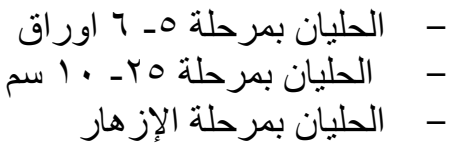

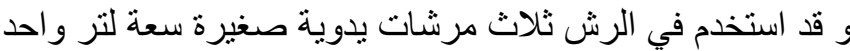

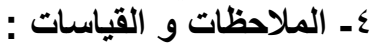

ا - تحديد كفاعة المبيدات المختبرة: على عثبة الحليان خلال مر احل النمو السابقة وذللك من

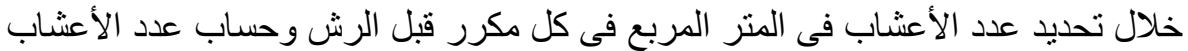

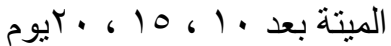

r- تأثير المكافحة الكيميائية على الإنتاجية: حيث تم حساب الإنتاجية فى كل مكرر ولكل تركيز من المبيدات المستخدمة. r- الجدوى الاقتصادية: قيمة المحصول - التكلفة الإجمالية = الربح الصـافى لكل معاملة وكل

Fayoum J. Agric. Res. \& Dev., Vol.22, No.2, July, 2008 


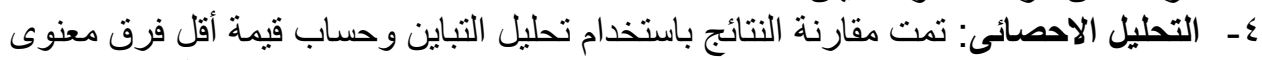

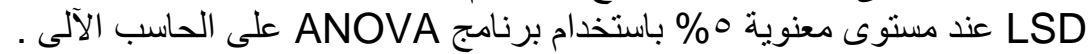
النتائج والمناقثشة

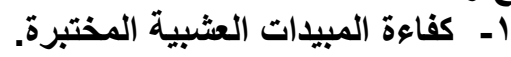

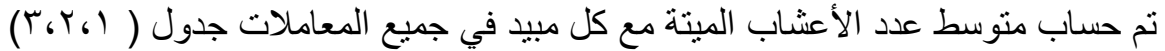

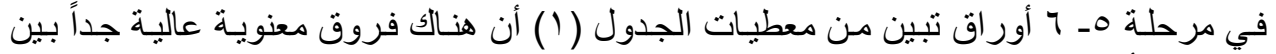

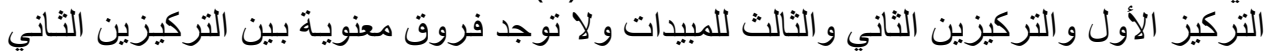

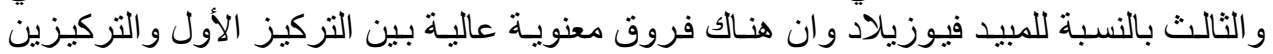

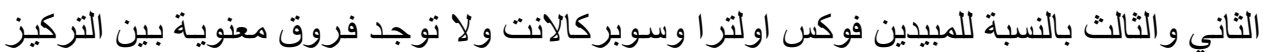

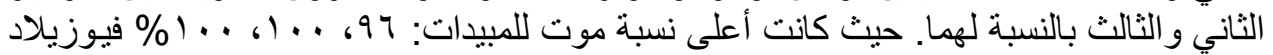

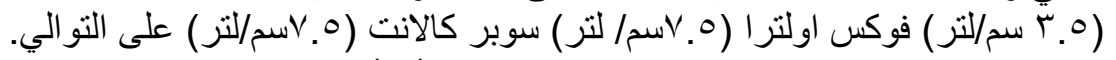

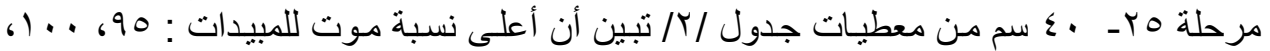

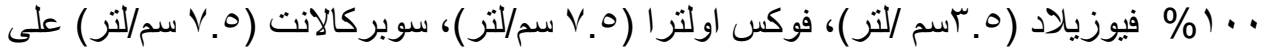

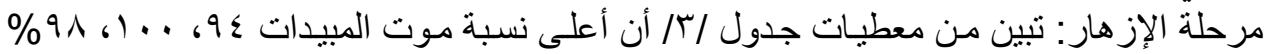

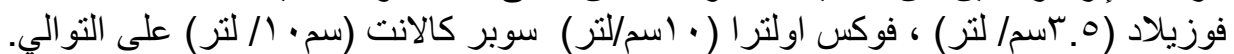

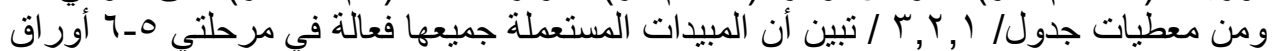

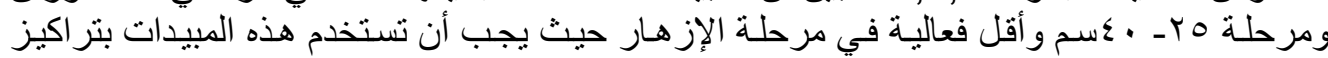
أعلى. (Corbett et al., 1994; Floyd et al., 1991)

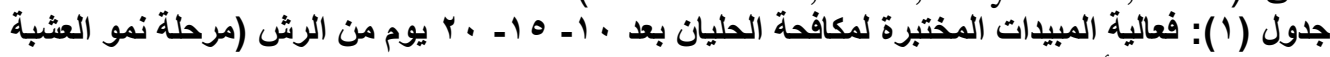

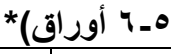

\begin{tabular}{|c|c|c|c|c|c|c|c|c|}
\hline \multirow{2}{*}{\multicolumn{2}{|c|}{ بعد ·rيوم }} & \multirow{2}{*}{\multicolumn{2}{|c|}{ بعد 10 يوم }} & \multirow{2}{*}{\multicolumn{2}{|c|}{ بعد • 1 أيام }} & \multirow{3}{*}{ الألعشئ قبّل } & \multirow{3}{*}{ سمدل المبيد استخام } & \multirow[b]{3}{*}{ المبيا } \\
\hline & & & & & & & & \\
\hline $\begin{array}{c}\text { \% } \\
\text { \% }\end{array}$ & الأعشاب علد & $\begin{array}{c}\text { \% للموت } \\
\text { \% }\end{array}$ & متوسطظ عدد الاعشيّة & $\begin{array}{c}\text { \% } \\
\text { \% }\end{array}$ & متوسط الأعشاب & & & \\
\hline 10 & IV & A. & 17 & 70 & 14 & $r$. & 1.20 & \multirow{3}{*}{$\begin{array}{c}\text { Fusillade } \\
25 \% \mathrm{EC}\end{array}$} \\
\hline $9 \leqslant$ & Tr & $\Lambda \wedge$ & TI & $V Y$ & ro & ro & $r .7$ & \\
\hline 97 & Tr & 91 & YY & $V$. & IV & $T \xi$ & r.o & \\
\hline $9 V$ & To & 94 & Tr & 10 & $r$. & T & 0 & \multirow{3}{*}{$\begin{array}{c}\text { Focus } \\
\text { ultra } 10 \% \mathrm{EC}\end{array}$} \\
\hline $1 \ldots$ & rA & 97 & $T V$ & NT & Tr & rA & $V .0$ & \\
\hline $1 \ldots$ & $\varepsilon \leqslant$ & 91 & $\varepsilon r$ & $\Lambda \varepsilon$ & $r v$ & $\varepsilon \varepsilon$ & 1. & \\
\hline 97 & rq & $9 \xi$ & rA & NT & YT & $\Gamma$. & 0 & \multirow{3}{*}{$\begin{array}{c}\text { Super gallant } \\
12.5 \% \mathrm{EC}\end{array}$} \\
\hline $1 \ldots$ & $\varepsilon Y$ & $9 \wedge$ & «1 & Ar & $\Gamma \varepsilon$ & $\varepsilon Y$ & Vo & \\
\hline $1 \ldots$ & $\leqslant 1$ & $1 \ldots$ & $\leqslant \Lambda$ & A. & rᄉ & $\leqslant 1$ & 1. & \\
\hline . & - & . & & · & - & YI & رش بالماء & الثـاهد \\
\hline
\end{tabular}

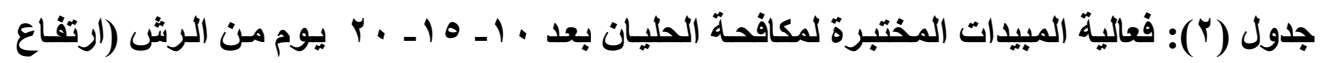

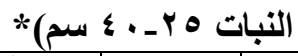

\begin{tabular}{|c|c|c|c|c|c|c|c|}
\hline \multicolumn{2}{|c|}{ 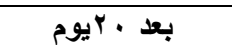 } & \multicolumn{2}{|c|}{ بعد 10 يوم } & \multicolumn{2}{|c|}{ بعد · 1 أيام } & \multicolumn{2}{|l|}{ معدل استخام متوسط عدد } \\
\hline \begin{tabular}{|l|l|} 
للموت \\
$\%$
\end{tabular} & متوسط عدد & $\begin{array}{l}\text { اللموت } \\
\text { \% }\end{array}$ & الأعثاب الميتّة & $\begin{array}{l}\text { اللموت } \\
\text { \% }\end{array}$ & الأعثاب الميتّة & 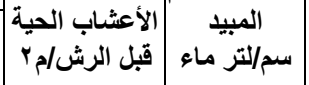 & المبيا \\
\hline
\end{tabular}

Fayoum J. Agric. Res. \& Dev., Vol.22, No.2, July, 2008 
$\varepsilon$

\begin{tabular}{|c|c|c|c|c|c|c|c|c|}
\hline & الميتة & & & & & & & \\
\hline$\lambda 1$ & $r 7$ & AI & ry & 07 & 11 & Tr & 1.10 & \multirow{3}{*}{$\begin{array}{c}\text { Fusillade } \\
25 \% \mathrm{EC}\end{array}$} \\
\hline $9 \pi$ & $\varepsilon r$ & 19 & $\leqslant 1$ & OY & $r \varepsilon$ & $\sum 7$ & Y. & \\
\hline 90 & YI & 91 & $r$. & 7. & 15 & TY & r. & \\
\hline 90 & r & 91 & ו ו & $V V$ & $r t$ & r & 0 & \multirow{3}{*}{$\begin{array}{c}\text { Focus } \\
\text { ultra } 10 \% \mathrm{EC}\end{array}$} \\
\hline $1 \ldots$ & $\varepsilon$. & 91 & rq & Vo & r. & $\varepsilon$. & $V .0$ & \\
\hline $1 \ldots$ & rᄉ & $1 \ldots$ & $r \wedge$ & $V V$ & rq & "ᄉ & 1. & \\
\hline 97 & ro & qr & $r \xi$ & 70 & 18 & $r 7$ & 0 & \multirow{3}{*}{$\begin{array}{c}\text { Super } \\
\text { gallant } \\
12.5 \% \mathrm{EC}\end{array}$} \\
\hline $1 \ldots$ & rᄉ & $1 \ldots$ & rA & $7 \leq$ & 11 & $r \wedge$ & $V .0$ & \\
\hline $1 \ldots$ & 0. & $1 \ldots$ & 0. & 01 & rq & 0. & 1. & \\
\hline - & . & . & - & - & - & rᄉ & رش بالماء & النّاهد \\
\hline
\end{tabular}

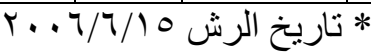

LSD $0.05=3.65$

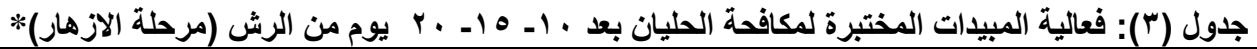

\begin{tabular}{|c|c|c|c|c|c|c|c|c|}
\hline \multicolumn{2}{|c|}{ بعد · بيوم } & \multicolumn{2}{|c|}{ بعد 10 يوم } & \multicolumn{2}{|c|}{ بعد · 1 أيام } & \multirow{2}{*}{ 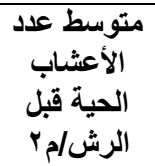 } & \multirow{2}{*}{ 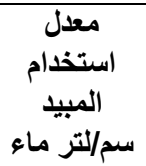 } & \multirow[b]{2}{*}{ المبيد } \\
\hline $\begin{array}{c}\text { للموت } \\
\text { \% }\end{array}$ & متوسط عدد الأعشاب & تلمو & متوسط عدد الأعشاب & $\begin{array}{c}\text { للموت } \\
\text { \% }\end{array}$ & متوسط عدد الأعشاب & & & \\
\hline VA & Tr & Vo & YI & $\varepsilon r$ & Ir & rA & 1.10 & \multirow{3}{*}{$\begin{array}{c}\text { Fusillade } \\
25 \% \mathrm{EC}\end{array}$} \\
\hline$\Lambda V$ & Y & NT & ro & $\varepsilon r$ & $1 \pi$ & r. & Y. & \\
\hline $9 \leq$ & r. & 9. & rV & \&1 & T & Tr & r.o & \\
\hline 9. & 19 & 19 & 11 & $7 Y$ & Tr & YI & 0 & \multirow{3}{*}{$\begin{array}{c}\text { Focus } \\
\text { ultra } 10 \% \mathrm{EC}\end{array}$} \\
\hline $9 V$ & ס & $9 r$ & س & 07 & $r$. & Tו & $V .0$ & \\
\hline $1 \ldots$ & rE & 97 & Tr & $0 \wedge$ & $1 \varepsilon$ & $T \varepsilon$ & 1. & \\
\hline$\wedge \wedge$ & rr & $\wedge \wedge$ & rr & r & $\Lambda$ & ro & 0 & \multirow{3}{*}{$\begin{array}{c}\text { Super } \\
\text { gallant } \\
12.5 \% \mathrm{EC}\end{array}$} \\
\hline $9 V$ & אי & $9 \varepsilon$ & Tr & ס & IT & Tะ & $V .0$ & \\
\hline 91 & rq & 90 & T人 & r. & Ir & $\varepsilon$. & 1. & \\
\hline 0 & - & - & 0 & - & 0 & 11 & رش بالماء & الشـاهد \\
\hline
\end{tabular}

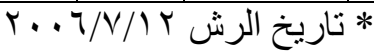

LSD0.05=4.18

r- بأثير المكافحة الكيميائية على الإنتاجية:

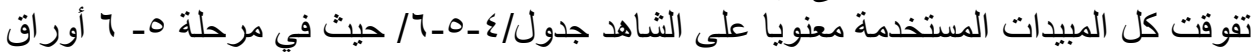

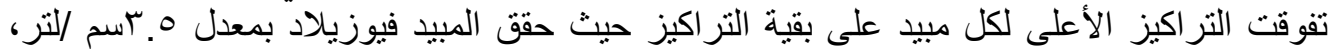

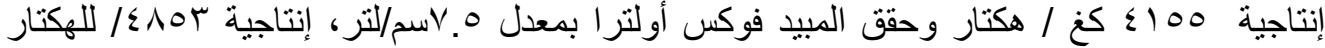

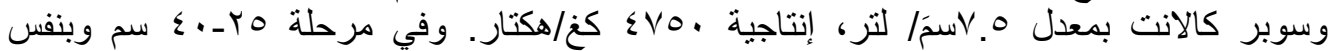

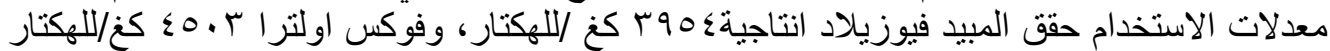

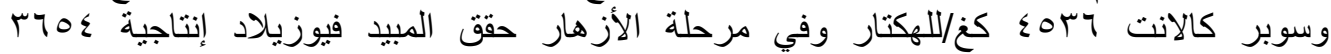

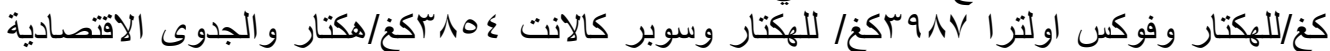

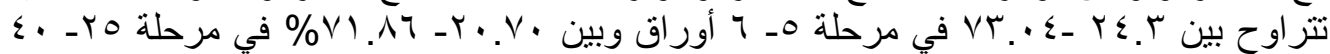

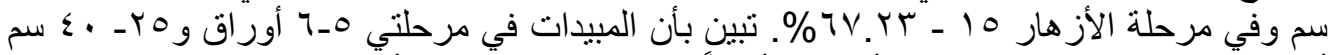

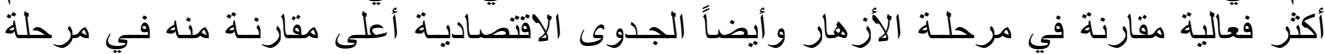
الأزهار .(Keazney et al, 1975).

Fayoum J. Agric. Res. \& Dev., Vol.22, No.2, July, 2008 
جدول (؛): تأثير المكافحة الكيميائية على الإتتاجية والجدوى الاقتصادية (الرش في مرحلة هـ 1 أوراق).

\begin{tabular}{|c|c|c|c|c|c|c|c|}
\hline الاقتصادية & لـس / الربح الصافي & لل للمحصر الإجمالي & لـس / كبر المبيع & 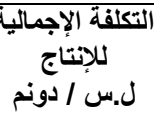 & كمية الإنتاج & | معدل استخدام & المبيد \\
\hline$r \varepsilon . r$. & $1 \ldots 0$ & $\leqslant 1 T 0$ & ro & ॉाr. & 170.5 & 1.10 & \multirow{3}{*}{$\begin{array}{c}\text { Fusillade } \\
25 \% \mathrm{EC}\end{array}$} \\
\hline $77.9 V$ & $7 \leq 0$. & 974. & ro & ए人. & r^o.r & $Y .7$ & \\
\hline 71.9. & VIOV & I.rNT & ro & TrT. & $\leqslant 10.0$ & r.o & \\
\hline 79.01 & VTr. & 1.0Y. & TO & r... & $\leqslant Y \cdot \Lambda$ & 0 & \multirow{3}{*}{$\begin{array}{c}\text { Focus } \\
\text { ultra } 10 \% \mathrm{EC}\end{array}$} \\
\hline VY.A. & ANTY.O & ITIKY & TO & ק & $\leqslant \wedge 0 . \mu$ & V.o & \\
\hline$V Y . \cdot T$ & $\wedge V \vee \cdot$ & IYIV. & ro & एะ.. & $\sum \wedge \uparrow . \wedge$ & 1. & \\
\hline $79 . \wedge \mathrm{V}$ & $V 19 Y$ & $1 . r 94$ & TO & M... & $\varepsilon 11 . V$ & 0 & \multirow{3}{*}{$\begin{array}{c}\text { gallant Super } \\
12.5 \% \mathrm{EC}\end{array}$} \\
\hline$V T . \leqslant V$ & AVYo & $111 \mathrm{VO}$ & TO & T10. & $\varepsilon \vee 0$ & $V .0$ & \\
\hline$V T . \cdot \varepsilon$ & ATV. & $11 \wedge V$. & ro & rr.. & $\leq V \leq .9$ & 1. & \\
\hline & OVO & TrVo & ro & r^.. & 1To & & الشاهد \\
\hline
\end{tabular}

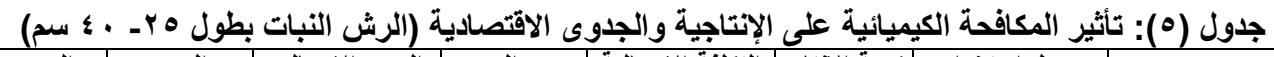

\begin{tabular}{|c|c|c|c|c|c|c|c|}
\hline الاقتصادية الجدوى & ل الص الربيح دوني & لل للسعر الإجمالي & لس / كعز المبيع & 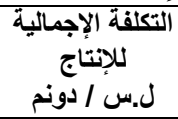 & كيّا دونم الآتتاج & سمل المبيد استخدام & المبيد \\
\hline$r \cdot . V$ & $\Lambda V .0$ & $r q \leqslant V .0$ & To & $\mu r$. & $10 \% .9$ & 1.10 & \multirow{3}{*}{$\begin{array}{c}\text { Fusillade } \\
25 \% \mathrm{EC}\end{array}$} \\
\hline $70 . Y$ & 097. & $91 \leqslant$. & To & T) & r70.7 & Y. 7 & \\
\hline $7 V . r$ & 7700 & 9110 & ro & rTr. & r90.5 & $r .0$ & \\
\hline $7 \wedge . \wedge$ & $V .7$. & $1 . \times 7$. & ro & r... & $\varepsilon 1 \cdot . \varepsilon$ & 0 & \multirow{3}{*}{$\begin{array}{c}\text { Focus } \\
\text { ultra } 10 \% \mathrm{EC}\end{array}$} \\
\hline$v \cdot .7 \wedge$ & V90V.0 & llrov.0 & ro & r... & $\leqslant 0 . r$ & $Y .0$ & \\
\hline$V \cdot . Y Y$ & $1.1 V .0$ & $11 \leqslant 1 V .0$ & ro & rะ.. & $\leqslant 07 . V$ & 1. & \\
\hline 79.19 & $V \cdot r$. & $1.1 \%$. & ro & M... & $\varepsilon .0 . Y$ & 0 & \multirow{3}{*}{$\begin{array}{c}\text { Super } \\
\text { gallant } \\
12.5 \% \mathrm{EC} \\
\end{array}$} \\
\hline VY.YY & 119. & 11Tद. & ro & Mlo. & sor. 7 & V.0 & \\
\hline$\vee 1 . \wedge 7$ & AIVY.O & IIKVY.O & ro & Tr.. & $\leqslant 0 \leqslant .9$ & 1. & \\
\hline & Tro & MITo & ro & rA.. & Iro & & الشـاهد \\
\hline
\end{tabular}

جدول (†): تأثير المكافحة الكيميائية على الإنتاجية والجدوى الاقتصادية (الرش في مرحلة الأزهار)

\begin{tabular}{|c|c|c|c|c|c|c|c|}
\hline $\begin{array}{c}\text { الاقتصادية } \\
\text { \% }\end{array}$ & ل الصافي دونم & لل للمحصول الإجمالي & لسر/ كبغ & للتلإلتاجة الإمالية & كمية الإنتاج & سمدل التزبيد معلخام & المبيد \\
\hline 10 & oor.o & rqAr.o & ro & rা & $1 \leqslant V . r$ & 1.20 & \multirow{3}{*}{$\begin{array}{c}\text { Fusillade } \\
25 \% \mathrm{EC}\end{array}$} \\
\hline $00 . \leqslant 7$ & r97. & $v) \leqslant$. & TO & TוA. & YA0.7 & T.7 & \\
\hline $7 \leq .7 \varepsilon$ & 09.0 & 9150 & To & TrT. & r70.5 & $r .0$ & \\
\hline 70.91 & T)AV.0 & 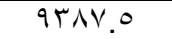 & TO & r... & rVo.0 & 0 & \multirow{3}{*}{$\begin{array}{c}\text { Focus } \\
\text { ultra } 10 \% \mathrm{EC}\end{array}$} \\
\hline 77.19 & ง TV.0 & $997 \% .0$ & TO & rr.. & rq৯.V & $V .0$ & \\
\hline 77.99 & $79 \ldots$ & $1 . r \ldots$ & TO & एะ.. & हार & 1. & \\
\hline EY.rY & TrVO & Orvo & ro & rI... & $r 10 . \varepsilon$ & 0 & \multirow{3}{*}{$\begin{array}{c}\text { Super gallant } \\
12.5 \% \mathrm{EC}\end{array}$} \\
\hline TV.r & $7 \leqslant 10$ & 9740 & ro & r10. & rAo.s & $V .0$ & \\
\hline TV.YT & $707 \mathrm{~V} .0$ & $9 \vee 7 \vee .0$ & ro & r... & $r q \cdot . v$ & 1. & \\
\hline & $r \ldots$ & $r .10$ & TO & $r \wedge \ldots$ & $1 Y \cdot .7$ & & الشاهد \\
\hline
\end{tabular}

خامساً. الاستنتاجات: من خلال در اسة تأثثر ثناثة مبيدات مختلفة على عشبة الحليان فى مر احل النمو

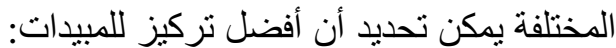

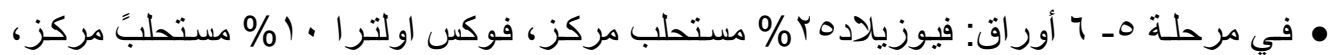

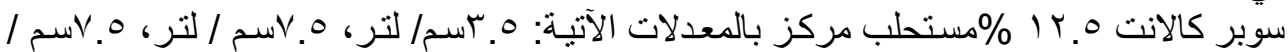

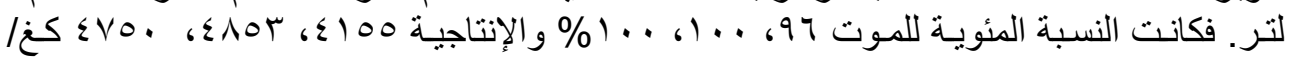

Fayoum J. Agric. Res. \& Dev., Vol.22, No.2, July, 2008 


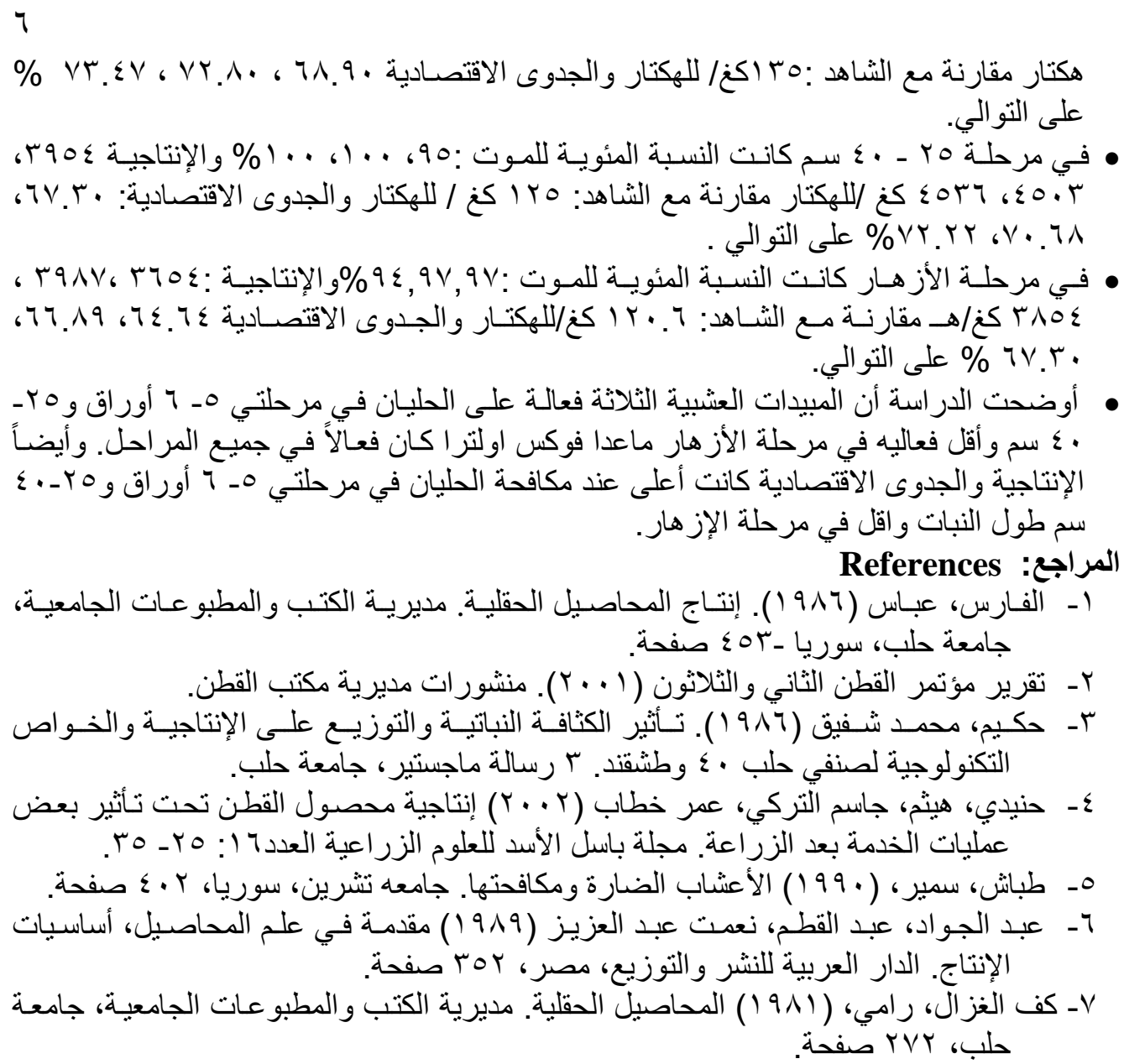

8- F.A.0 (1991). Integrated pest management. instructions on cotton insect pests. Vol. 7. Rome, Italy.

9 - F.A.O (1996). Year book production Vol. 80 . Rome, Italy.

10- Corbett, J.R.; Wright, K. and Baillie, A.C. (1994). The biochemical mode of action of pesticides, second edition, Academic press, London . $282 \mathrm{pp}$.

11- Flayd, M. A.; Alben, S. C. (1991). Mod of action of herbicides. A Wiley, Intersection Publication, New York, Chichester Brisbane Toronto, 525 pp.

12- Keazney, P.C.; Kaufman, D.D. (1975). Herbicides : Chemistry, degradation and mode of action, $2^{\text {nd }}$ edition, Revised and expanded, Vol. 1, Marcel Dekker INC, New York and Basel, 500 pp.

A COMPARISON OF THE EFFICIENCY OF SOME HERBICIDES FOR CONTROLLING SORGHUM HALEPENSE (L.) IN COTTON FIELDS IN DIFFERENTS GROWTH PERIODS AND THE EFFECT ON PRODUCTIVITY.

\section{Asawad Al- Mhemid}

Crop Protection Dept. - Faculty of Agriculture, University of Al- Furat- Syria 


\section{ABSTRACT}

A field study was carried out to compare the efficiency of three herbicides in three concentrations for controlling Sorghum halepense (L.) in three periods of plant development.

Results showed superior effects of herbicides :

* In 5 - 6 leaves period:

For Fusilade 25\% EC Focus ultra 10\%EC Super Gallant $12.5 \%$ EC in the concentration of $3.5 \mathrm{~cm}^{3} / \mathrm{L} ., 7.5 \mathrm{~cm}^{3} / \mathrm{L}, 7.5 \mathrm{~cm}^{3} / \mathrm{L}$ the mortality percentages were: $96,100,100 \%$ and roductivity $4155,4853,4750 \mathrm{~kg} / \mathrm{h}$ in comparison with $135 \mathrm{~kg} / \mathrm{h}$ for control as with economic useifulness: 68.90, $72.80,73.47 \%$, respectively.

* In $25-40 \mathrm{~cm}$ period:

Mortality percentages were: $95,100,100 \%$ and productivity: 3954, 4503, $4536, \mathrm{~kg} / \mathrm{h}$ in comparison with control $(125 \mathrm{~kg} / \mathrm{h})$ economic usefulness: $67.30,70.68: 72.22 \%$, respectively.

*In Florescence period:

Mortality percentages were: $94,97,97 \%$ and productivily: 3654,3987 , $3854 \mathrm{~kg} / \mathrm{h}$ in comparison with control $(120.6 \mathrm{~kg} / \mathrm{h})$ and Economically usefulness: 64. 64., 66 . 89:67.30\% respectively.

Study showed that three herbicides were effective against Sorghum halepense (L.) In 5-6 leaves and $25-40 \mathrm{~cm}$ periods and less effective in the Florescence period Focusultra was effective in all of periods.

Also productivity and economic usefulness were higher with Sorghum halepense control in 5-6 leaves and 25-40 cm period and less effective in Florescence period.

Key words: Herbicides: Fusilade, Focusultra Super Gallant-Sorghum halepense (L.)

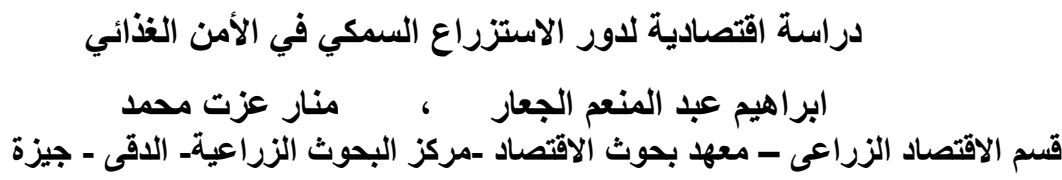

Fayoum J. Agric. Res. \& Dev., Vol.22, No.2, July, 2008 
$\wedge$

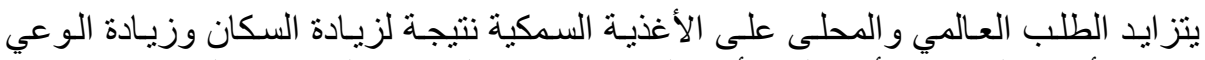

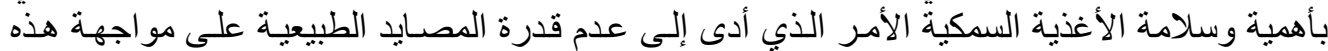

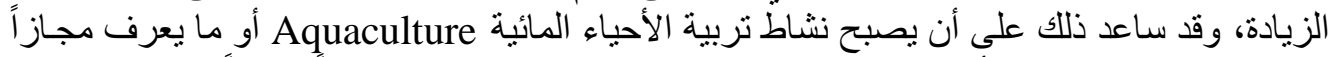

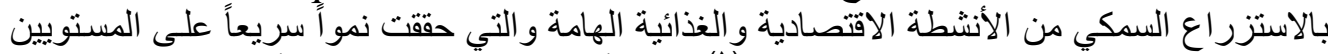

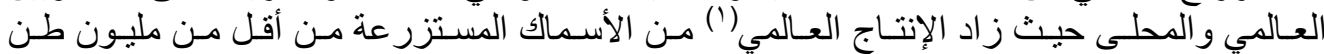

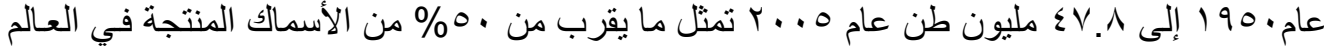
من المصايد الطبيعية المبية

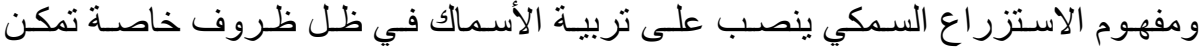

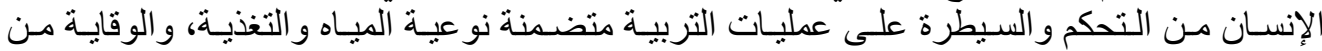

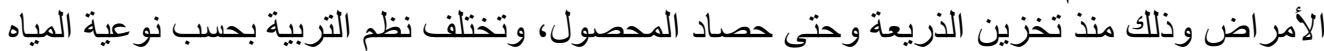
(عذبه - مالحةـ شروب) وبحسب معدل تكثيف الأنغ الأسماك المرباة (مكثفه أو شبه مكثفه).

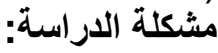

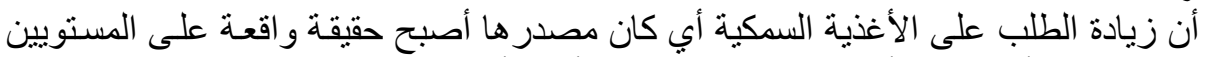

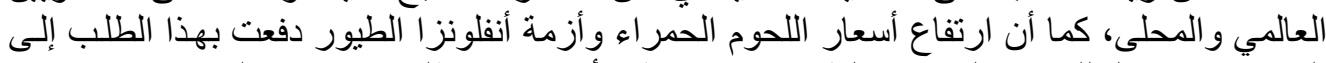

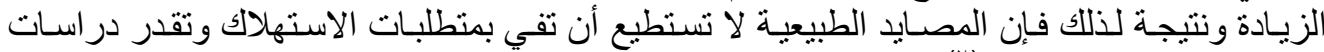

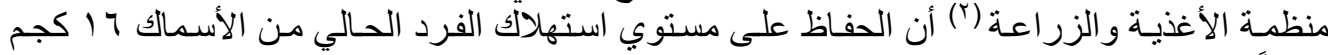

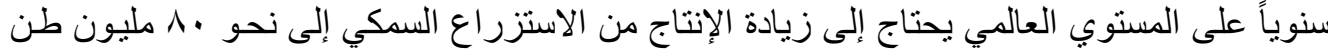

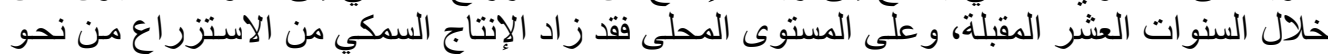

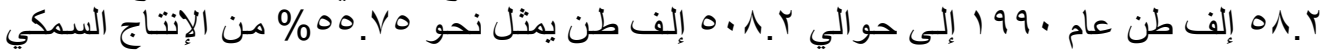

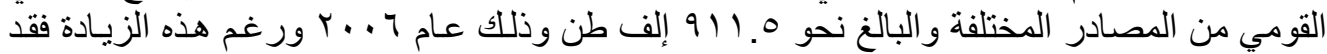

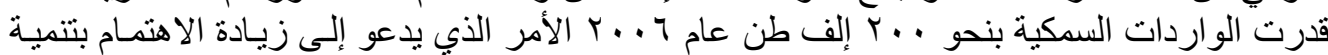

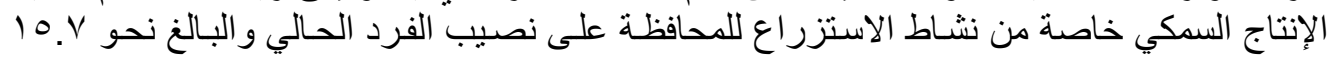

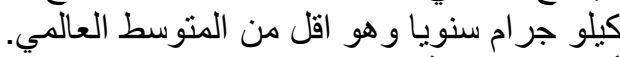

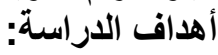

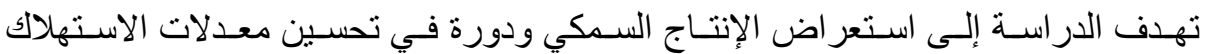

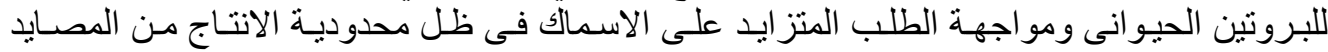

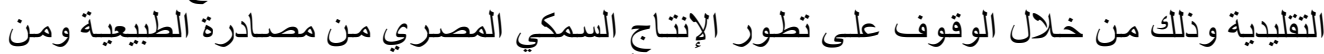

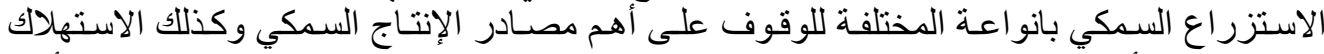

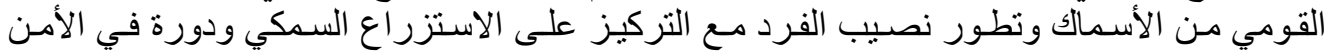

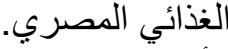

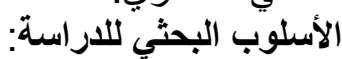

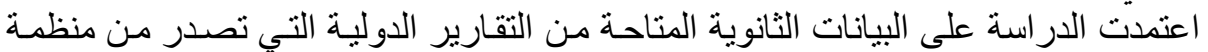

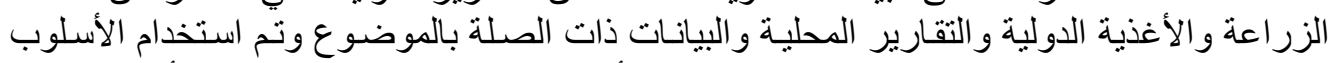

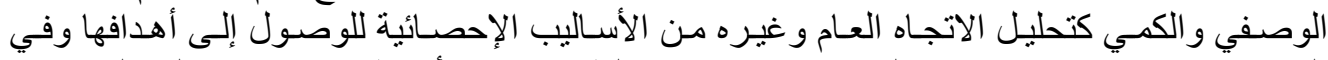

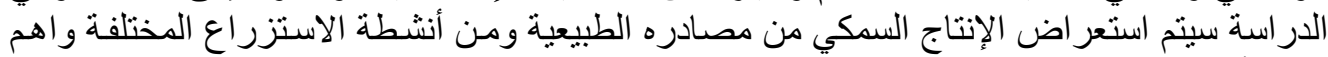

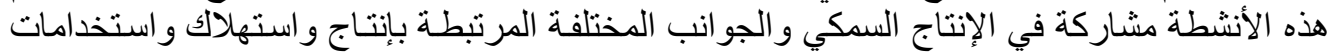

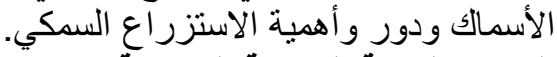

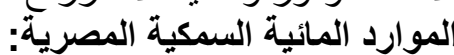
تنقسم الموارد المائية السمكية إلى قسمين:

1) FAO. Fisheries Department, State OF Word aquaculture ,2006

") منظمة الأغذية والزر اعة، التقرير الإقليمي للشرق الأدنى وشمال أفريقيا عن الاستزراع السمكي، روما r...

Fayoum J. Agric. Res. \& Dev., Vol.22, No.2, July, 2008 
الأول الموارد أو المصادر الطبيعية أو مايطلق عليه المصايد الطبيعية ممثلة في البحرين الأبيض

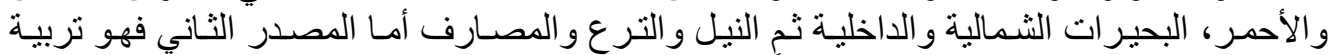

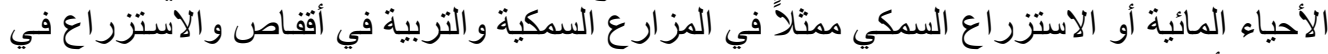

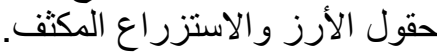

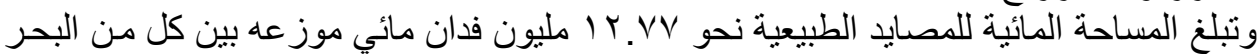

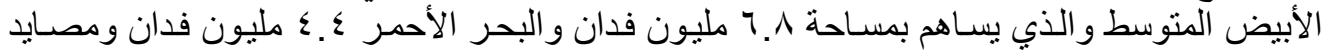

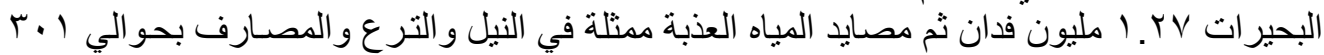

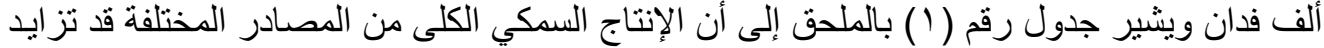

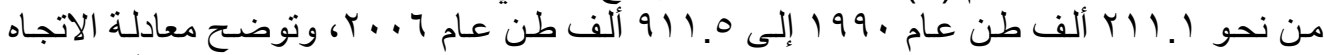

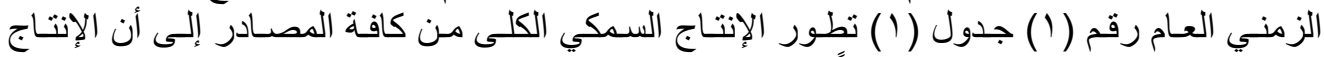

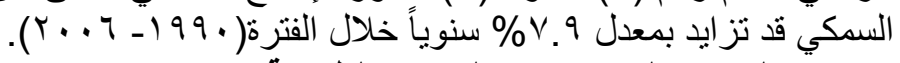

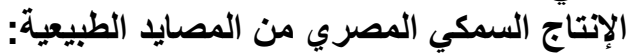

تشمل المصايد الطبيعية المصايد الطبيعية البحرية ممثلة في البحرية البيدية المنتوسط و البحر الأحمر

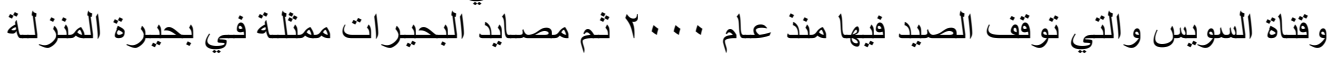

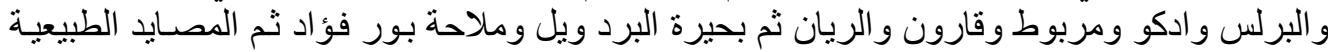

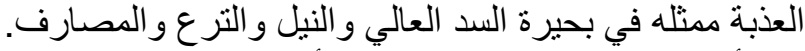
أ - إنتاج البحرين المتوسط والأحمر النول

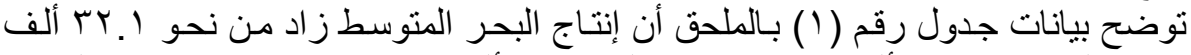

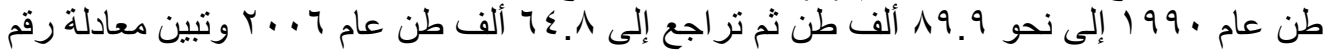

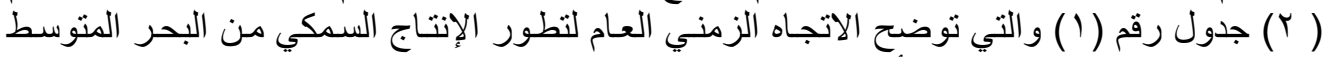

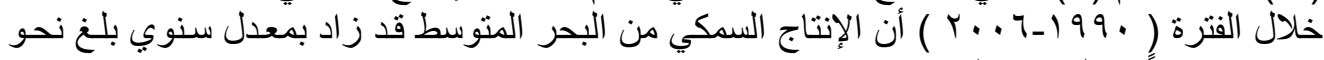

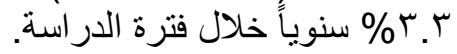

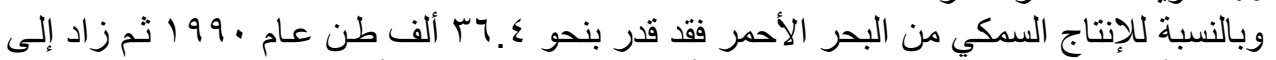

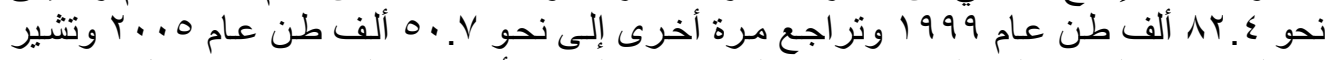

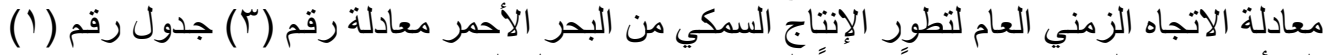

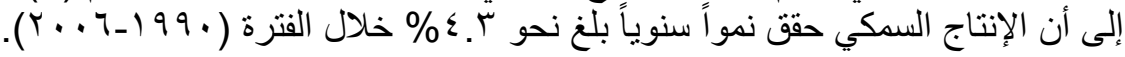

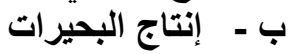

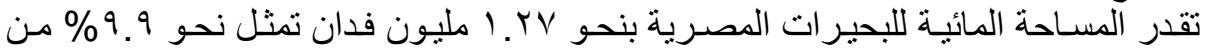

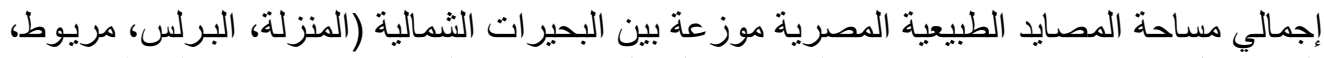

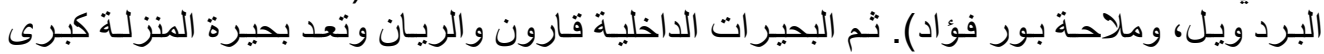

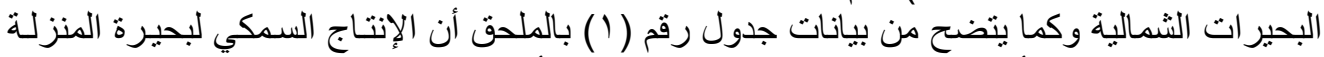

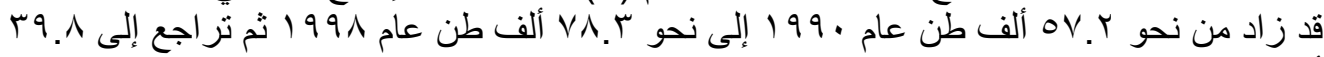

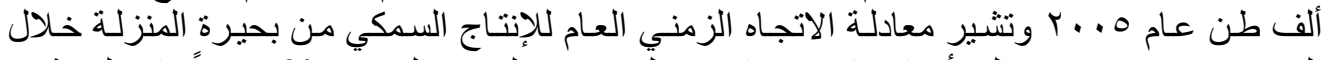

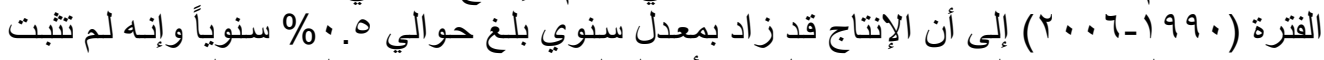

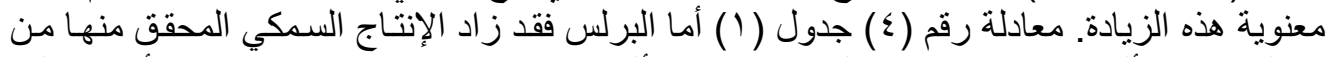

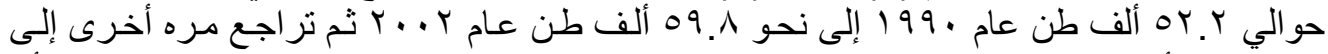

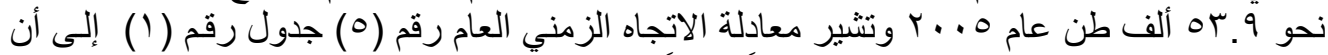

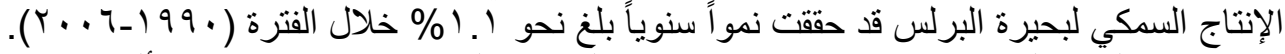

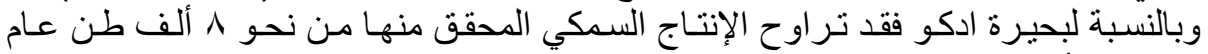

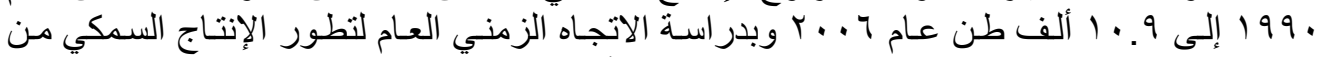

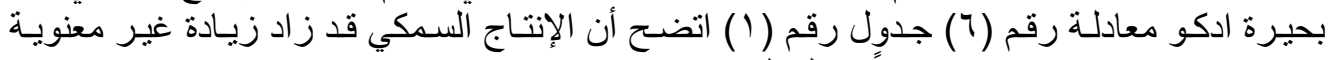

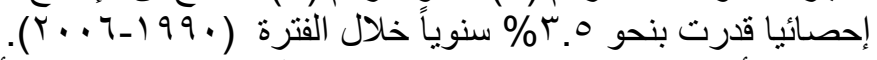

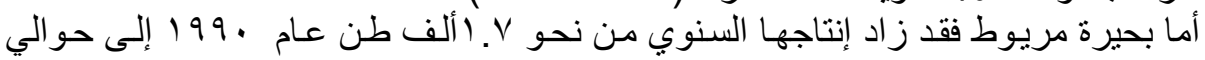

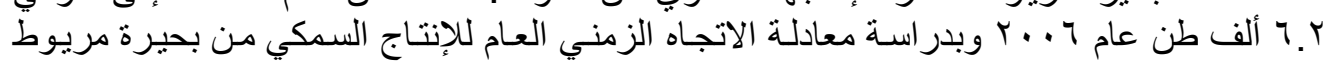

Fayoum J. Agric. Res. \& Dev., Vol.22, No.2, July, 2008 
1.

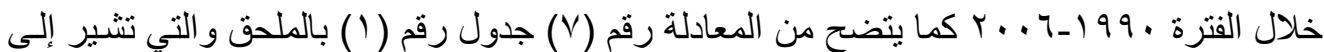

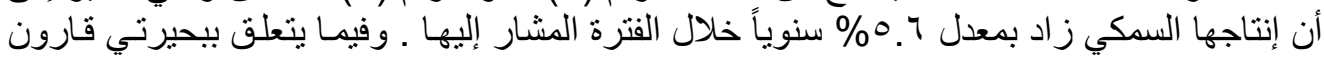

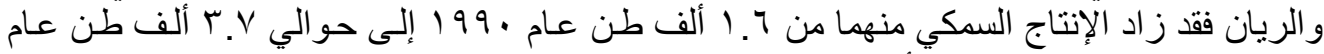

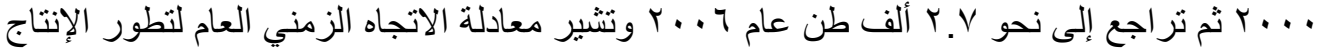

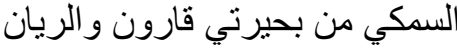
جدول رقم (1) الاتجـاه الزمنـي العـام لتطور الإنتاج السمكي من المصـايد الطبيعية المصرية خلال

\begin{tabular}{|c|c|c|c|c|c|c|}
\hline المعنوية & $\mathbf{F}$ & $\mathbf{R}^{\overline{2}}$ & $\mathbf{R}^{2}$ & المعادلة & البيان & المعادلة \\
\hline معنوى & plq.rq & .90 & .99 & $\begin{array}{r}Y=261.44 e^{0.079} \\
(Y \vee . \vee \wedge)\end{array}$ & الاجمالى & 1 \\
\hline معنوى & $11 . r 1 \%$ &..$r q$ & $\because \varepsilon r$ & 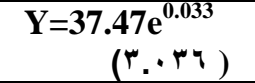 & البحرالمتوسط & $r$ \\
\hline معنوى & YV.O. &. $.7 Y$ & $\because .7 \leqslant$ & $\begin{array}{r}Y=37.87 e^{0.043} \\
\left(\bullet . Y^{\circ}\right)\end{array}$ & البحر الاحمر & $r$ \\
\hline غير معنوى & 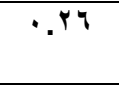 & $-\cdot \cdot \leqslant \Lambda$ & $\because \cdot 1 \mathrm{~V}$ & $\begin{array}{r}Y=55.23 \mathrm{e}^{0.005} \\
(\cdot .01)\end{array}$ & بحيرة المنزلة & $\varepsilon$ \\
\hline غير معنوى & 7.79 & .94 & $\cdot r$. & $\begin{array}{c}Y=49.32 \mathrm{e}^{0.011} \\
(\Upsilon . \diamond \wedge)\end{array}$ & بحيرة البرلس & 0 \\
\hline غير معنوى & r.YV & .10 & .19 & $\begin{array}{r}\mathrm{Y}=6.27 \mathrm{e}^{0.035} \\
(1.9 \mu)\end{array}$ & بحيرة ادكو & 7 \\
\hline غير معنوى & 17.75 & $\because \leqslant \leqslant$ & $\because \leqslant V$ & $\begin{array}{r}Y=2.63 e^{0.056} \\
(Y .79)\end{array}$ & بحيرة مريوط & V \\
\hline غير معنوى & $r . \cdot v$ & $.1 Y$ &. $.1 \mathrm{~V}$ & $\begin{array}{c}Y=1.54 e^{0.032} \\
(1 . \vee \bullet)\end{array}$ & بحيرة البردويل & $\wedge$ \\
\hline غير معنوى & $7.1 \mathrm{~V}$ & $\because r \leq$ & $\because r q$ & $\begin{array}{r}Y=1.75 \mathrm{e}^{0.038} \\
(Y . \leqslant \Lambda)\end{array}$ & بحيرة قارون & 9 \\
\hline معنوى & Y.V. & $\because .97$ & $\because 10$ & $\begin{array}{r}\mathrm{Y}=0.21 \mathrm{e}^{-0.024} \\
(-1.7 \varepsilon)\end{array}$ & بحيرة بور فؤاد & 1. \\
\hline معنوى & 79.74 &.$\wedge 1$ & $\because \wedge r$ & $\begin{array}{r}\mathrm{Y}=36.17 \mathrm{e}^{0.075} \\
(\wedge . \Psi \varepsilon)\end{array}$ & النيل والترع & 11 \\
\hline
\end{tabular}

المصدر : جمعت وحسبت من جدول ( (1) بالملحق

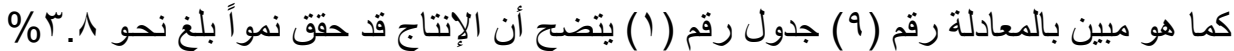

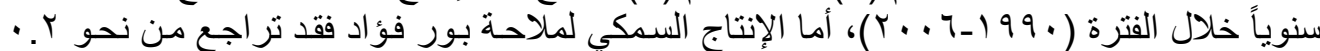

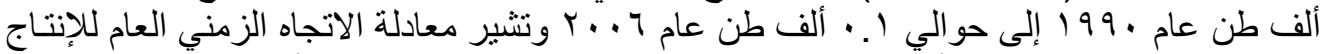

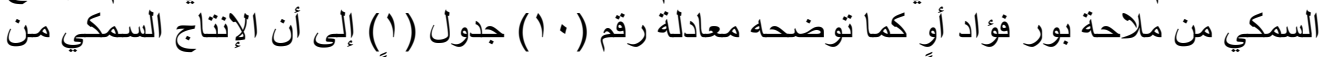

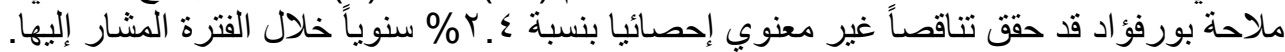

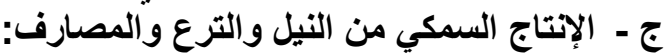

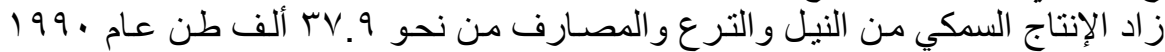

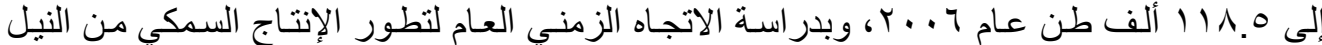

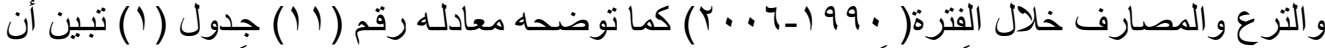

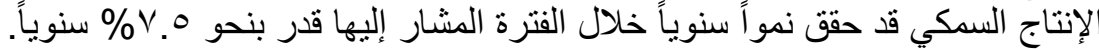

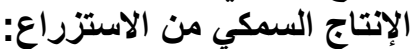

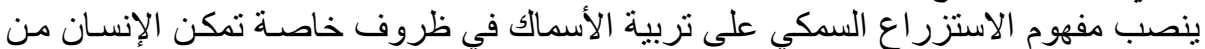

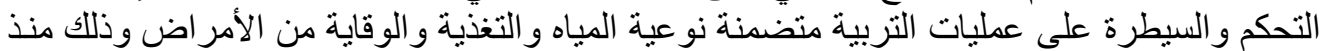

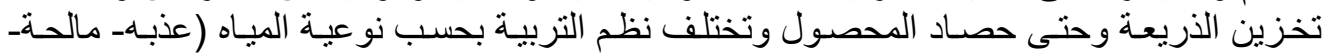
شروب) وبحسب معدل تكثيف الأسماك المر بـاه (مكثف أو شبه مكثف) وتعد صناعة الاستزراع

Fayoum J. Agric. Res. \& Dev., Vol.22, No.2, July, 2008 
11

السمكي (َّ) نو عاً من أنواع الإنتاج الزر اعي مثل الدحاصيل النباتية أو الحبو انبة فهي تلبـى طلبـاً غذائياً

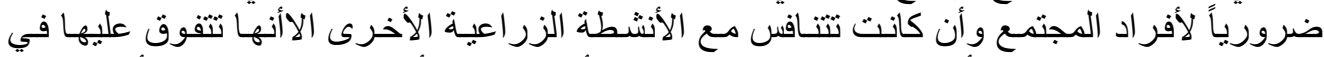

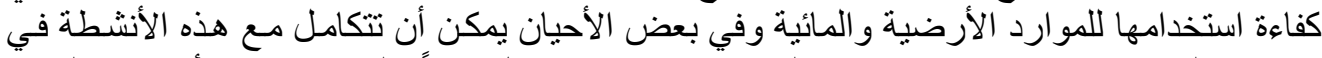

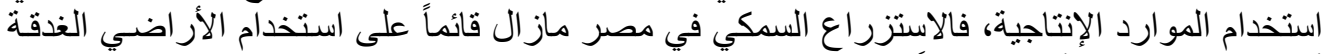

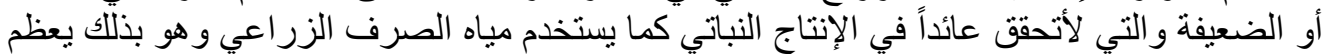

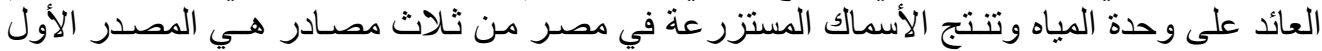

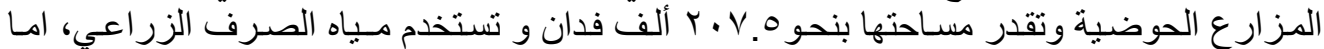

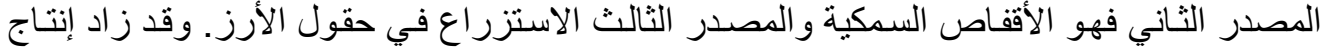

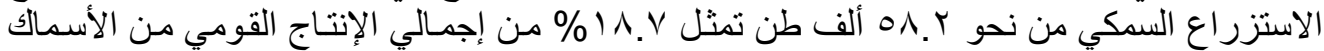

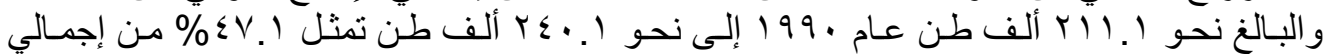

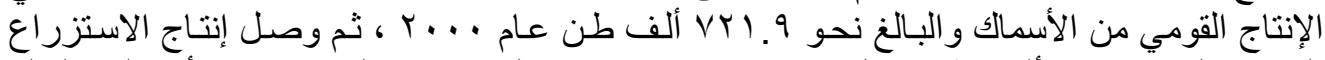

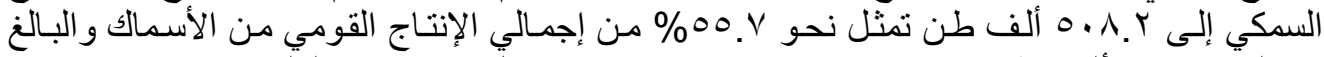

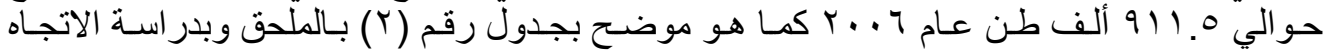

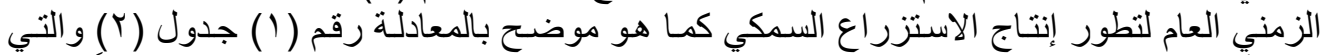

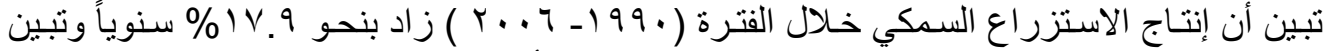

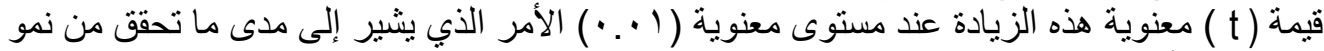

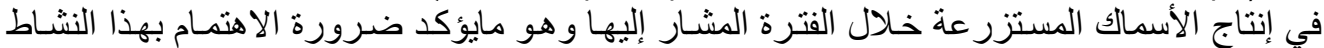

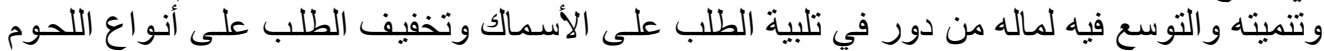

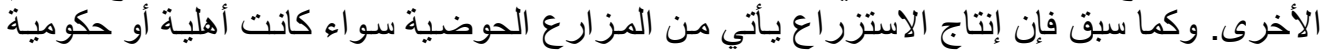

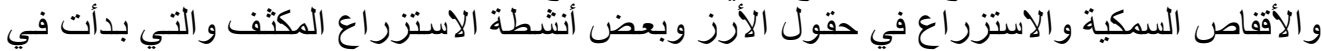

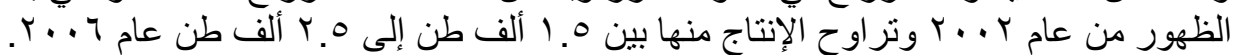

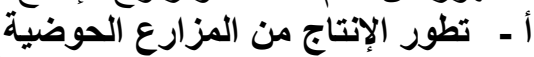

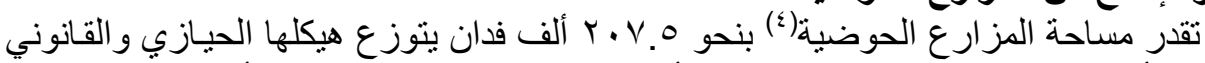

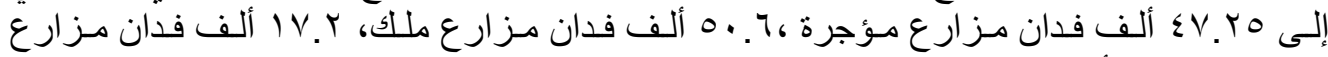

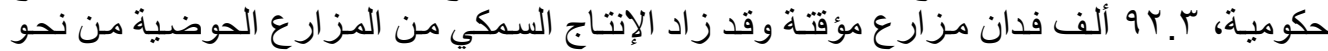

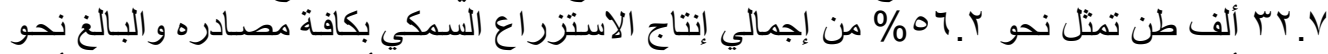

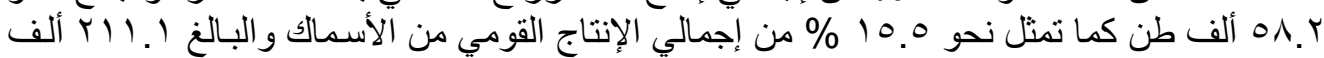

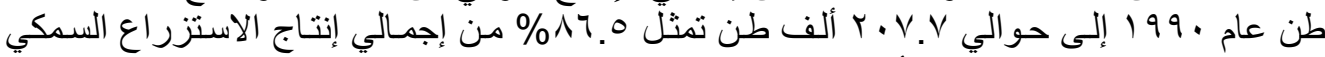

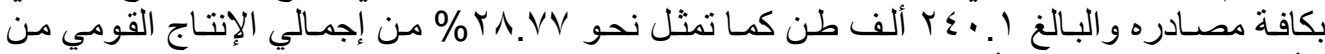

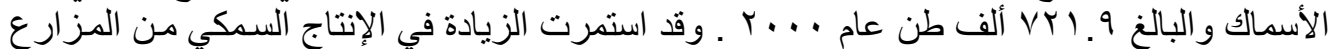

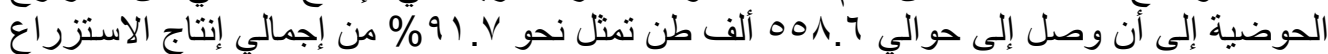

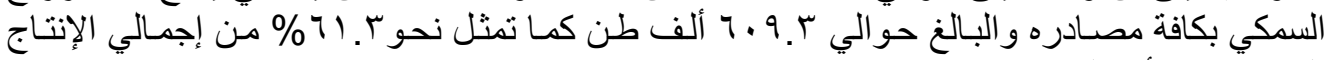

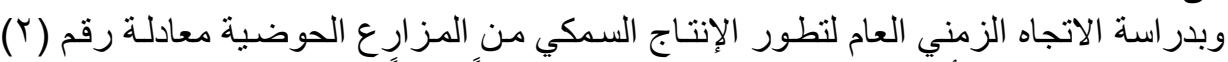

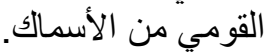

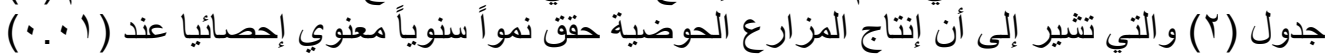

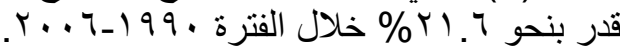

جدول رقم (ץ) الاتجاه الزمني العام لتطور الإنتاج السمكي من مصادر الاستزراع المختلفة خلال

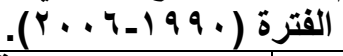

\begin{tabular}{|c|c|c|c|c|}
\hline $\mathbf{F}$ & $\mathbf{R}^{2}$ & رقم المعادلة & رالبيان \\
\hline
\end{tabular}

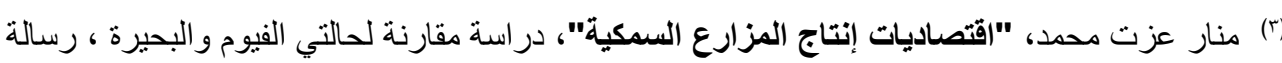

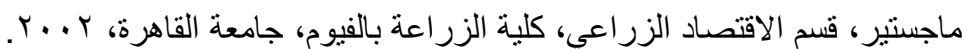

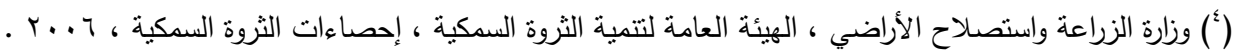

Fayoum J. Agric. Res. \& Dev., Vol.22, No.2, July, 2008 
it

\begin{tabular}{|c|c|c|c|c|}
\hline & $\cdot v$. & $\begin{array}{c}Y=36.61 e^{0.179} \\
(५ . \cdot \varepsilon)\end{array}$ & اجمالى & 1 \\
\hline $09 .+r$ &.$\vee v 9$ & $\begin{array}{c}Y=15.19 \mathrm{e}^{0.216} \\
(\vee . \neg \wedge)\end{array}$ & الحوضية & $r$ \\
\hline$\varepsilon \varepsilon .0$ & $\because v 0$ & $\begin{array}{c}Y=0.292 \mathrm{e}^{0.291} \\
(7.7 \varepsilon)\end{array}$ & اقفاص & $r$ \\
\hline $1 . \leqslant \varepsilon$ & $\because \cdot r q$ & $\begin{array}{c}Y=20.36 \mathrm{e}^{-0.019} \\
(-1.19)\end{array}$ & حقول الارز & \& \\
\hline
\end{tabular}

المصدر :جمت وحسبت من بيانات جدول (Y) بالملحق

ب - تطور الإنتاج من الأقفاص السمكية:

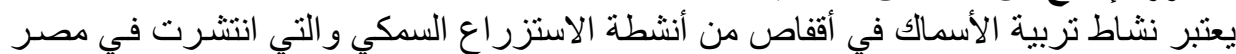

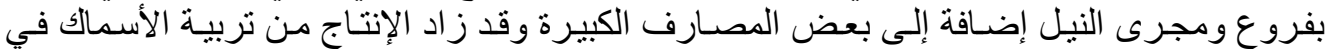

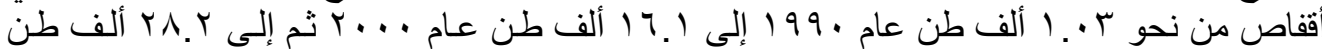

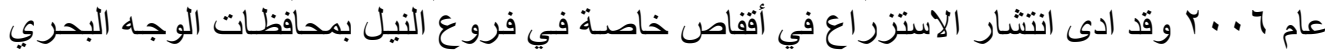

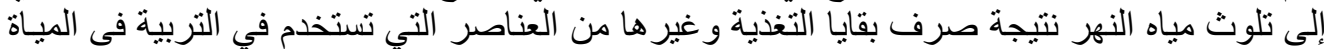

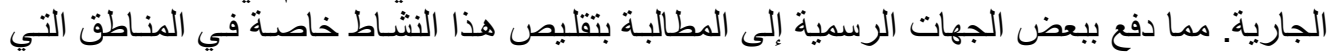
تعتبر مأخذ لمحطات تنقية مياه الثرب الترب.

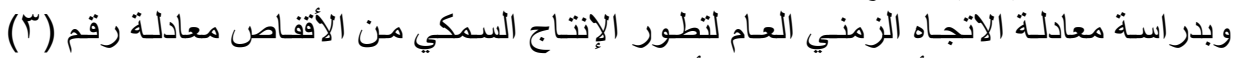

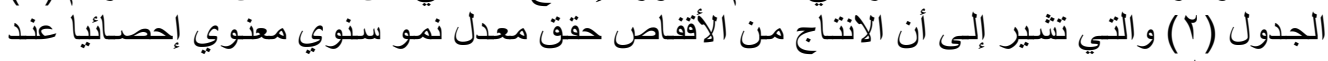

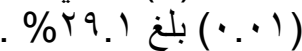
ج - تطور الإنتاج السمكي من حقول الأرز:

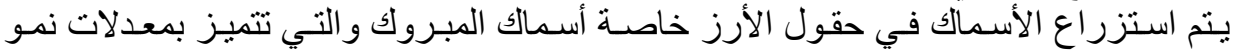

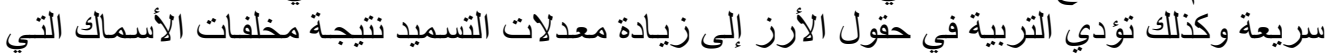

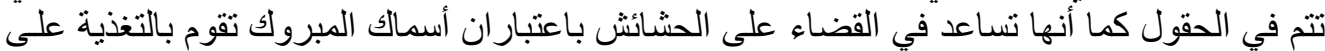

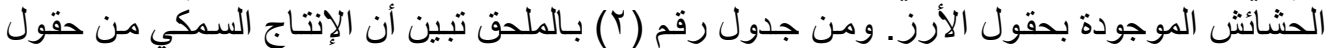

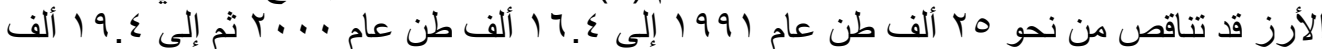

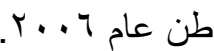

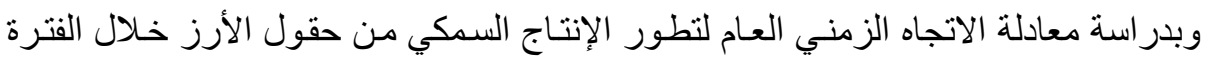

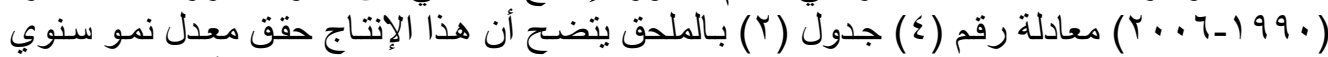

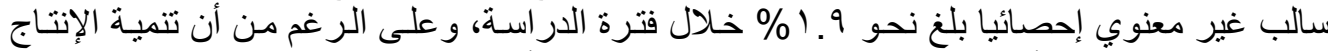

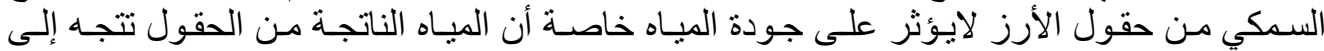

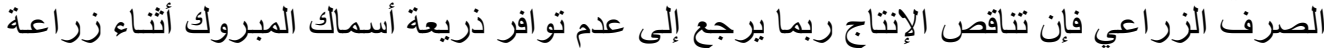
محصول الأرز الأمر الذي يحتاج إلى الاهتمام بذللك في السنوات التئ المقبلة.

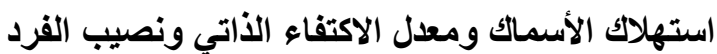

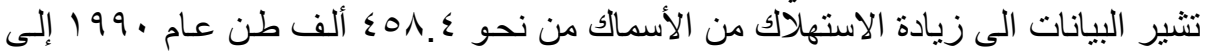

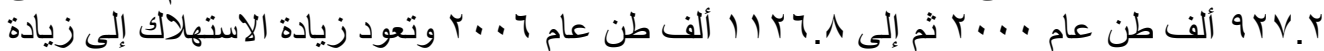

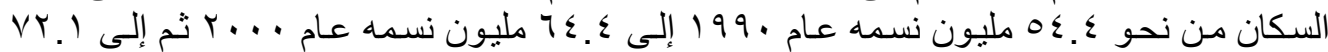

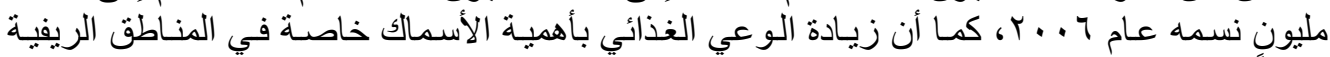

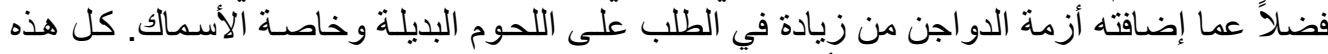

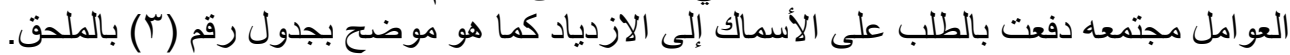

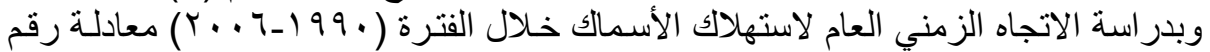

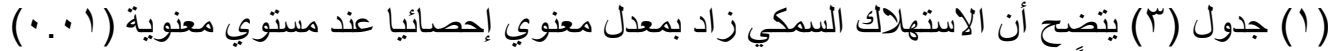

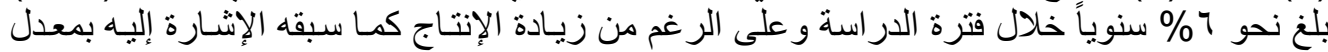

Fayoum J. Agric. Res. \& Dev., Vol.22, No.2, July, 2008 


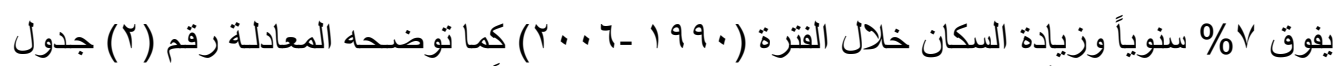

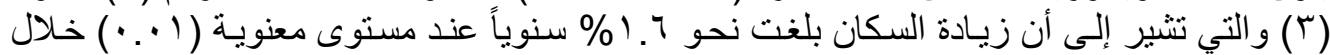

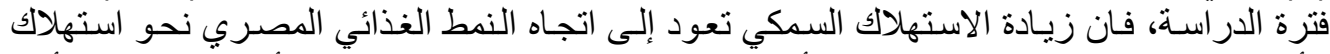

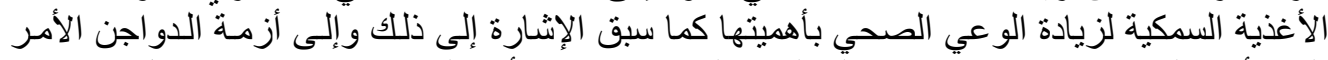

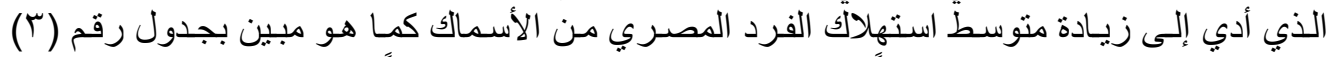

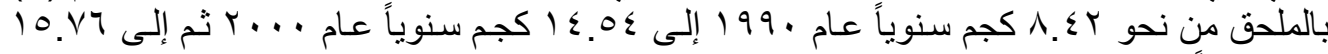

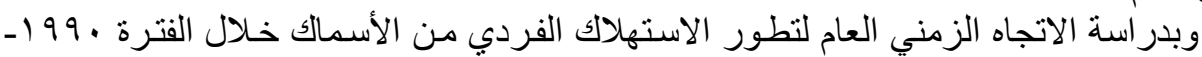

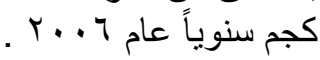

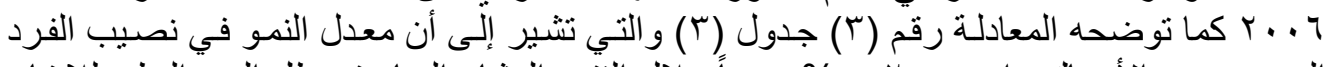

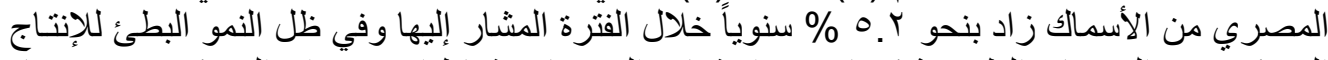

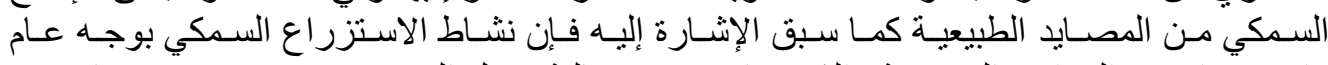

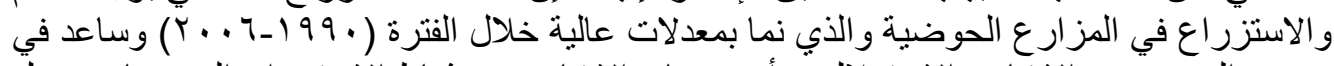

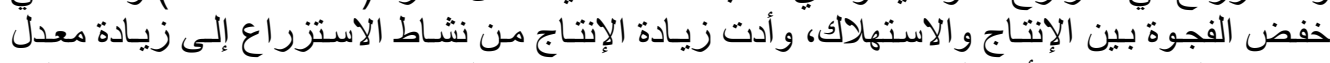

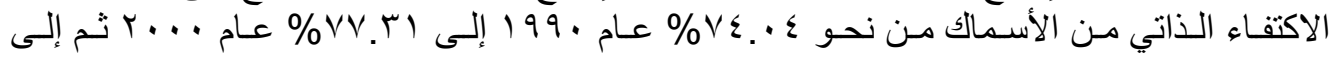

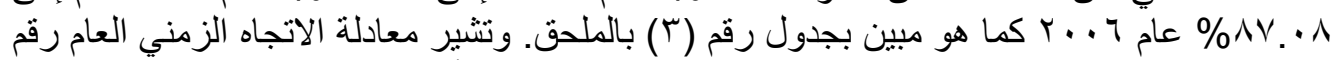

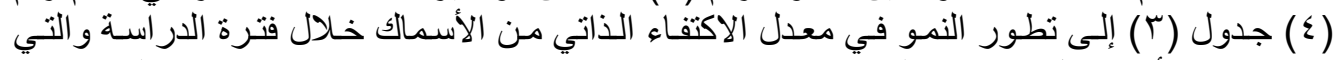

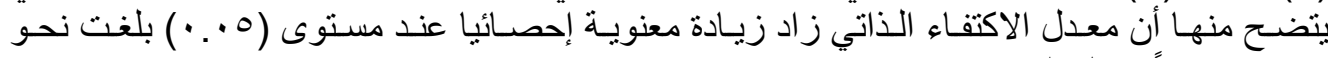

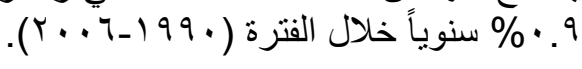

الواردات والصادرات السمكية:

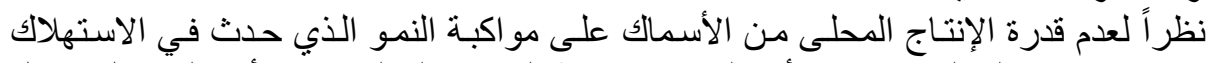

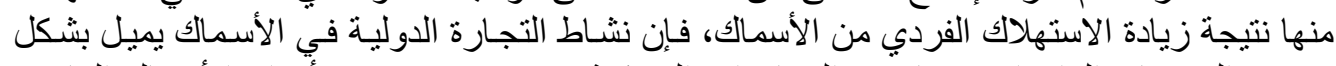

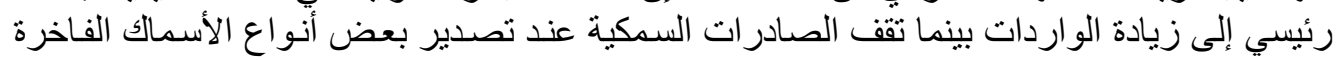

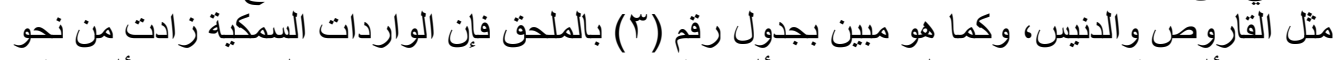

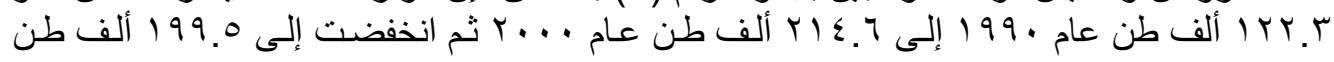

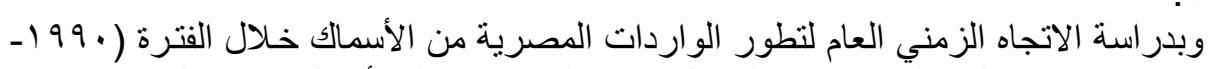

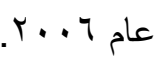

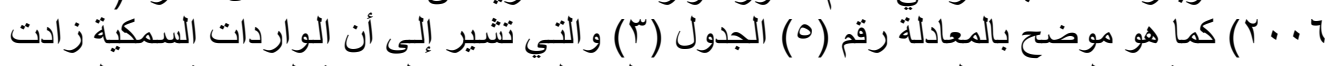

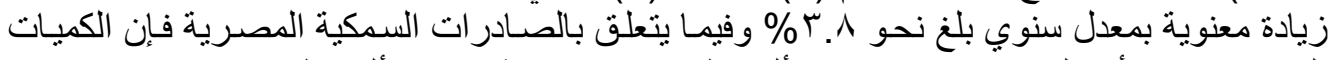

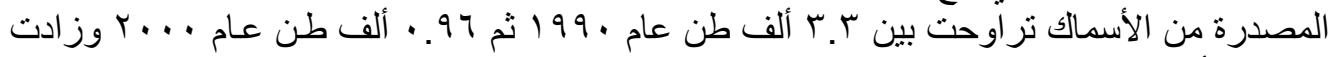

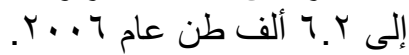

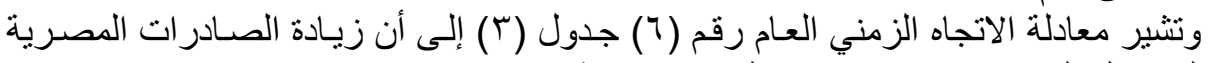

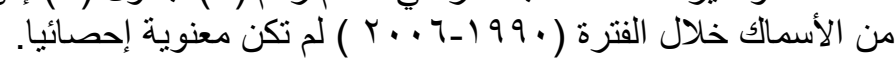

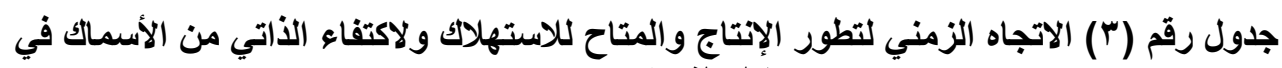

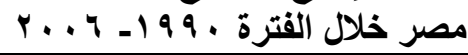

\begin{tabular}{|c|c|c|c|c|c|}
\hline $\mathbf{F}$ & $\mathbf{R}^{2}$ & $\mathbf{R}^{2}$ & المعادلة & البيان & رقم المعادلة \\
\hline MIA.Y & .90 & .90 & 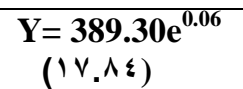 & استهلاك الاسماك & 1 \\
\hline $11 \leqslant \Lambda .0 \leqslant$ & .91 & .99 & $\begin{array}{c}\mathrm{Y}=53729.9 \mathrm{e}^{0.016} \\
(r r . \wedge q)\end{array}$ & السكان & r \\
\hline $178 . . \varepsilon$ & .91 & .94 & $\begin{array}{l}Y=7.24 e^{0.052} \\
(I Y . q Y)\end{array}$ & استهلاك فردى & $r$ \\
\hline $10 . r v$ & $\cdot . \leqslant V$ & $\because 0$ & $\begin{array}{c}Y=71.37 e^{0.009} \\
(ץ . q Y)\end{array}$ & معدل الاكتفاء الذاتى & $\varepsilon$ \\
\hline$r \cdot .77$ & .00 & $.0 \mathrm{~V}$ & $Y=115.83 e^{0.038}$ & الواردات & 0 \\
\hline
\end{tabular}

Fayoum J. Agric. Res. \& Dev., Vol.22, No.2, July, 2008 
$1 \leqslant$

\begin{tabular}{|c|c|c|c|c|c|}
\hline & & & $(\varepsilon .0 \leqslant)$ & & \\
\hline $1 . .1$ & $\because \cdots$ & $\therefore$ TV & $\begin{array}{l}Y=1.40 e^{0.034} \\
(1 . . \varepsilon)\end{array}$ & الصادرات & 7 \\
\hline
\end{tabular}

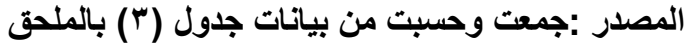

الملخص والتوصيات

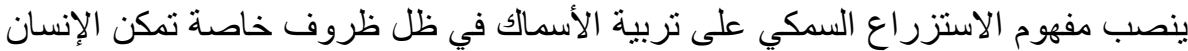

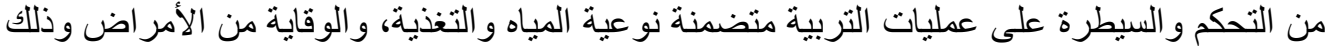

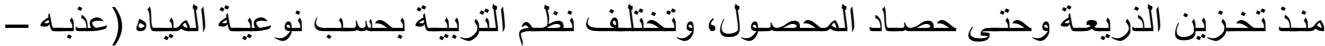

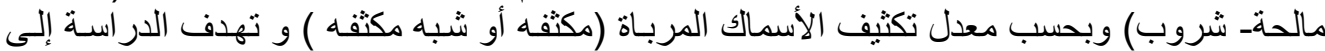

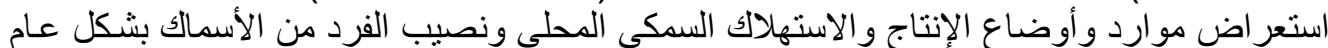

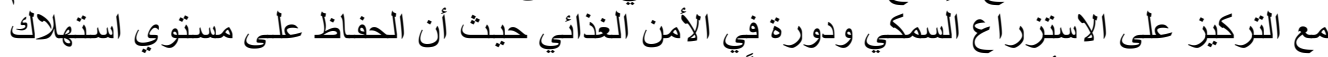

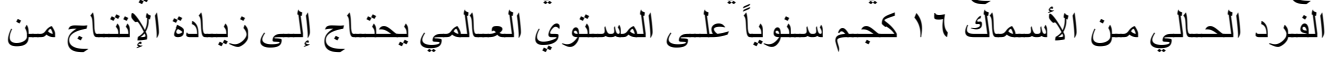

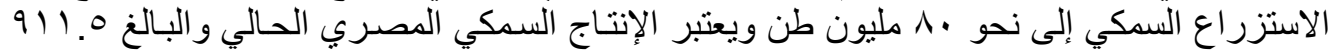

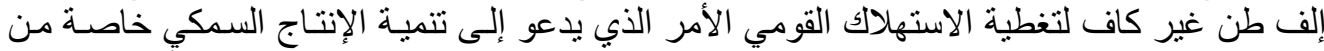

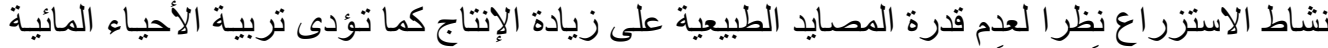

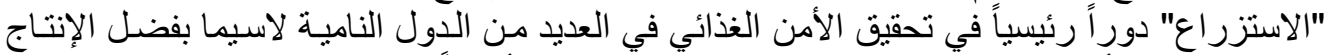

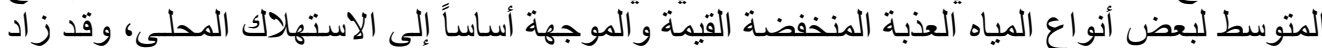

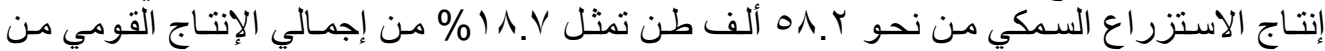

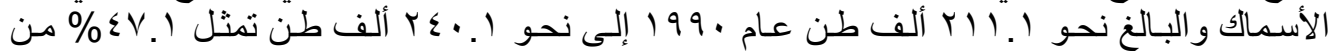

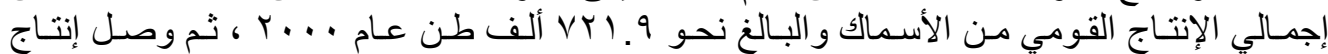

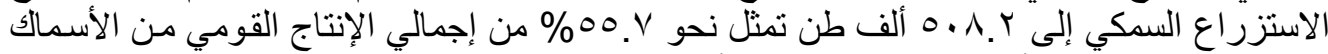

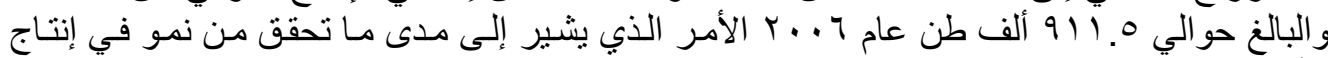

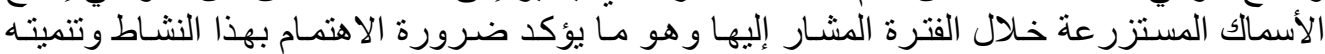

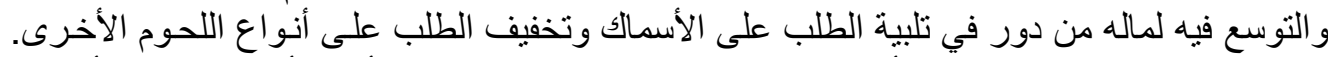

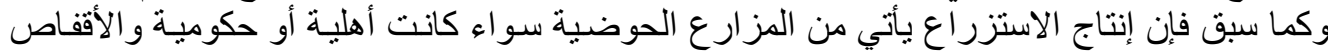

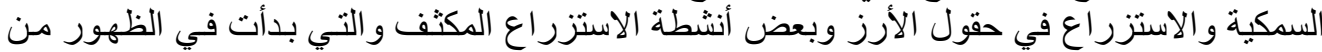

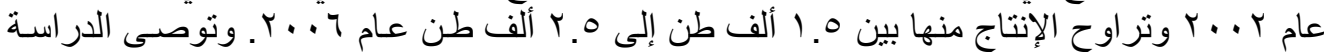

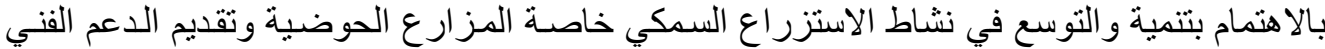

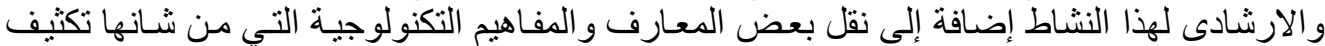

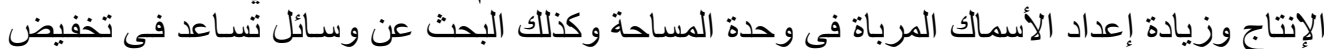

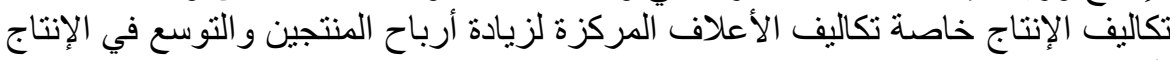

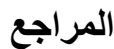

1. منظمة الأغذية والزر اعة الفاو، مصلحة مصايد الأسماك، حالة الموارد السمكية وتربية الأحياء

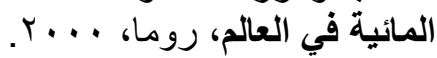

r. منظمة الأغذية والزر اعة الفاعة روماو، مصلحة مصايد الأسماك، حالة الموارد السمكية وتربية الأحياء

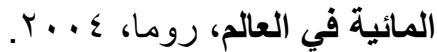

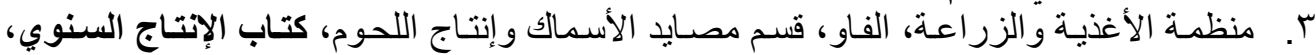

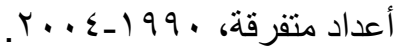

ع. منظمة الأغذية و الزر اعة الفاو، مصلحة مصايد الأسماك، حالة الموارد السمكية وتربية الأحياء

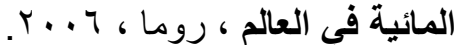

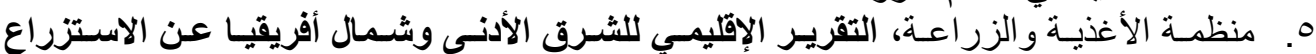

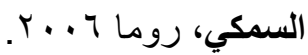

Fayoum J. Agric. Res. \& Dev., Vol.22, No.2, July, 2008 


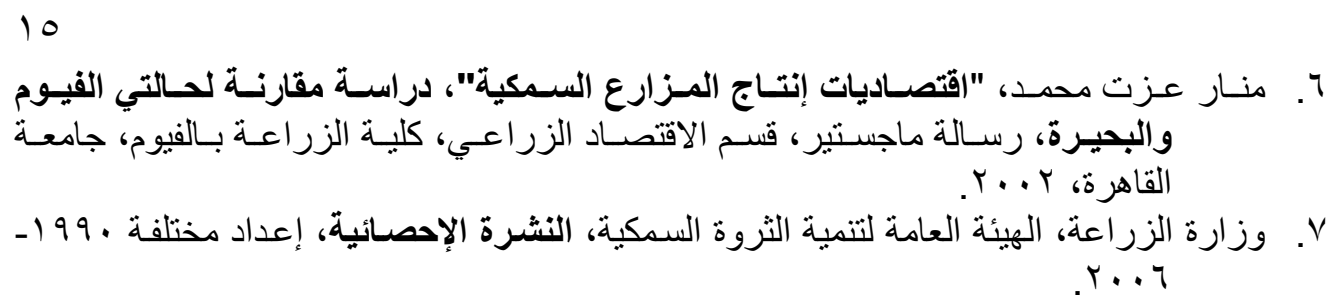

8. FAO. Fisheries Department, State oF Word Aquaculture, 2006.

\title{
An ECONOMIC STUDY IMPORTANCE ONAQUACULTURE IN FOOD SECURITY
}

\begin{abstract}
:
The study aims to explain the role of aquaculture and importance in the total production of fish at the global and local level

Aquaculture, probably the fastest growing food-producing sector, now accounts for almost 56 percent of the local food fish and is perceived as having the greatest potential to meet the growing demand for aquatic food, total aquaculture production Jumped from 58 thousand tones in 1990 to 508 thousand tones in 2006. The high rate of return on investment in aquaculture has attracted a large number of small to middle sized investors.

The study indicates, at different points that fish farming industry largely contributes to providing animal protein and to improving food selfsufficiency. In addition, it utilizes resources that are either unused or of low profitability when used for plant production, it usually exists in fallow, uses drainage water, and consequently maximizes the return from water (unit) and generates work opportunities and income for rural community.
\end{abstract}


Fayoum J. Agric. Res. \& Dev., Vol.22, No.2, July, 2008 
Fayoum J. Agric. Res. \& Dev., Vol.22, No.2, July, 2008 
11

Fayoum J. Agric. Res. \& Dev., Vol.22, No.2, July, 2008 